\title{
ALMOST TOTALLY COMPLEX POINTS ON ELLIPTIC CURVES
}

\author{
XAVIER GUITART, VICTOR ROTGER, AND YU ZHAO
}

\begin{abstract}
Let $F / F_{0}$ be a quadratic extension of totally real number fields, and let $E$ be an elliptic curve over $F$ which is isogenous to its Galois conjugate over $F_{0}$. A quadratic extension $M / F$ is said to be almost totally complex (ATC) if all archimedean places of $F$ but one extend to a complex place of $M$. The main goal of this note is to provide a new construction for a supply of Darmon-like points on $E$, which are conjecturally defined over certain ring class fields of $M$. These points are constructed by means of an extension of Darmon's ATR method to higher-dimensional modular abelian varieties, from which they inherit the following features: they are algebraic provided Darmon's conjectures on ATR points hold true, and they are explicitly computable, as we illustrate with a detailed example that provides numerical evidence for the validity of our conjectures.
\end{abstract}

\section{Contents}

1. Introduction

2. Quadratic points on modular abelian varieties

2.1. Quadratic extensions and $L$-functions

2.2. Oda-Yoshida's conjecture

2.3. Darmon points

2.4. Darmon-Logan's algorithm for the computation of ATR points

3. Almost totally complex points

3.1. Construction of ATC points

3.2. Comparison with Gartner's ATC points

3.3. A numerical example

\section{INTRODUCTION}

Let $E$ be an elliptic curve defined over a number field $F$ and, for any field extension $K / F$, let $L(E / K, s)$ denote the Hasse-Weil $L$-function of the base change of $E$ to $K$, which is known to converge on the half-plane $\left\{s \in \mathbb{C}: \operatorname{Re}(s)>\frac{3}{2}\right\}$.

The Mordell-Weil theorem asserts that the abelian group $E(K)$ of $K$-rational points on $E$ is finitely generated, that is to say,

$$
E(K) \simeq T \times \mathbb{Z}^{r},
$$

Received by the editors April 16, 2012 and, in revised form, May 28, 2012, October 2, 2012 and October 4, 2012.

2010 Mathematics Subject Classification. Primary 11G05, 11G40. 
where $T$ is a finite group and $r=r(E / K) \geqslant 0$ is a non-negative integer, which is called the Mordell-Weil rank of $E / K$.

There are two conjectures which stand out as cornerstones in the arithmetic of elliptic curves:

Conjecture (MOD). The elliptic curve $E / K$ is modular: there exists an automorphic representation $\pi$ of $\mathrm{GL}_{2}\left(\mathbb{A}_{K}\right)$ such that $L\left(E / K, s-\frac{1}{2}\right)=L(\pi, s)$. In particular, $L(E / K, s)$ can be analytically continued to an entire function on the complex plane and it satisfies a functional equation relating the values at $s$ and $2-s$.

Conjecture (BSD). Assume that (MOD) holds for $E / K$ and let $r_{a n}(E / K)=$ $\operatorname{ord}_{s=1} L(E / K, s)$ denote the order of vanishing of $L(E / K, s)$ at $s=1$, which we call the analytic rank of $E / K$. Then

$$
r(E / K)=r_{a n}(E / K) .
$$

Nowadays Conjecture (MOD) is known to hold, under mild hypothesis, when $F$ is totally real and $K / F$ is Galois with solvable Galois group, thanks to the work of Wiles, Skinner-Wiles, Langlands and others. More precisely, when $F$ is totally real, $E$ is known to be modular by [Wi], BCDT], SW], unconditionally if the base field is $F=\mathbb{Q}$ and under some technical conditions on the reduction type at the primes of $F$ above 3 when $[F: \mathbb{Q}]>1$. In this setting, the modularity of $E$ amounts to the existence of a Hilbert modular eigenform $f_{E}$ of parallel weight 2 over $F$ such that $L(E / F, s)$ is equal to the $L$-function $L\left(f_{E}, s\right)$ associated to $f_{E}$. If $K / F$ is solvable, then (MOD) follows from the modularity of $E$ over $F$ by applying Langlands's cyclic base change. If $F=\mathbb{Q}$ and $K$ is a totally real Galois number field, recent work of Dieulefait [Di] proves (MOD) under simple local assumptions on $K$, and one can expect that in the future similar techniques may lead to a similar result for arbitrary totally real fields $F$.

In light of these results, we assume throughout that $F$ is totally real and $E$ is modular. Let $N$ denote the conductor of $E$, an integral ideal of $F$, which for simplicity we assume to be squarefree.

Thanks to the work of Kolyvagin, Gross-Zagier and Zhang, Conjecture (BSD) is then known to hold when $K$ is either $F$ or a totally imaginary extension of $F$, $r_{a n}(E / K) \leqslant 1$ and the Jacquet-Langlands (JL) hypothesis holds:

$(\mathrm{JL})$ Either $[F: \mathbb{Q}]$ is odd or $N \neq(1)$.

In particular, when $K$ is a totally imaginary extension of $F$ and $r_{a n}(E / K)=1$, the above results imply that if (JL) is satisfied, there exists a non-torsion point in $E(K)$. Precisely when (JL) holds, such a point $P_{K}$, a so-called Heegner point, can be manufactured by means of the theory of complex multiplication on Shimura curves, and it is Gross-Zagier GZ and Zhang [Z] who showed that the hypothesis $r_{a n}(E / K)=1$ implies that $P_{K}$ is not torsion. Finally, Koyvagin's method [Ko] of Euler systems is the device which permits one to show that in fact there are no points in $\mathbb{Q} \otimes E(K)$ which are linearly independent of $P_{K}$, thereby showing (BSD). This is made possible thanks to the existence, along with the point $P_{K}$, of a system

$$
\left\{P_{c} \in E\left(H_{c}\right), c \geqslant 1,(c, \operatorname{disc}(K / F))=1\right\}
$$

of rational points on $E$ over the ring class field $H_{c} / K$, the abelian extension of $K$ associated by class field theory to the Picard group $\operatorname{Pic}\left(\mathcal{O}_{c}\right)$ of invertible ideals in the order $\mathcal{O}_{c} \subset K$ of conductor $c$ of $K$. 
That this supply of points should exist can be predicted using Conjecture (BSD), even if $K$ is not totally imaginary, as we now explain. Let $K / F$ be any quadratic field extension. Write

$$
N=N^{+} \cdot N^{-}
$$

as the product of two ideals $N^{+}, N^{-}$of $F$ where $N^{+}$(resp. $N^{-}$) is the product of the prime divisors of $N$ which split or ramify (resp. remain inert) in $K$.

A character $\chi: \operatorname{Gal}\left(K^{a b} / K\right) \rightarrow \mathbb{C}^{\times}$is called a ring class character if it corresponds, via the identification provided by class field theory, to a character of $\mathbb{A}_{K}^{\times}$whose restriction to $\mathbb{A}_{F}^{\times}$is trivial. They are precisely the characters factoring through $\operatorname{Gal}(H / K)$ for some ring class field $H$. Let $\chi: \operatorname{Gal}\left(K^{a b} / K\right) \rightarrow \mathbb{C}^{\times}$be a ring class character of finite order and conductor relatively prime to $N$. Let $r_{1}(K / F)$ and $r_{2}(K / F)$ be the number of archimedean places of $F$ which extend to a couple of real (resp. to a complex) place(s) of $K$, so that $[F: \mathbb{Q}]=r_{1}(K / F)+r_{2}(K / F)$. Then the sign of the functional equation of the $L$-function $L(E / K, \chi, s)$ of $E / K$ twisted by $\chi$ is

$$
\operatorname{sign}(E / K)=\operatorname{sign}(E / K, \chi)=(-1)^{r_{2}(K / F)+\sharp\left\{\wp \mid N^{-}\right\}},
$$

independently of the choice of $\chi$.

For any ring class field $H / K$, let $\widehat{\operatorname{Gal}}(H / K)=\operatorname{Hom}\left(\operatorname{Gal}(H / K), \mathbb{C}^{\times}\right)$denote the group of characters of $\operatorname{Gal}(H / K)$. The $L$-function of the base change of $E$ to $H$ factors as

$$
L(E / H, s)=\prod_{\chi \in \widehat{\operatorname{Gal}}(H / K)} L(E / K, \chi, s) .
$$

The Birch and Swinnerton-Dyer conjecture (BSD) in combination with (2) gives rise to the following conjecture.

Conjecture 1.1. Assume $\operatorname{sign}(E / K)=-1$ and let $H$ be a ring class field extension of $K$, unramified at the primes dividing $N$. Then

$$
\operatorname{rank} E(H)=[H: K]
$$

if and only if $L^{\prime}(E / K, \chi, 1) \neq 0$ for all $\chi \in \operatorname{Hom}\left(\operatorname{Gal}(H / K), \mathbb{C}^{\times}\right)$.

No proven result is known about Conjecture 1.1 beyond the achievements of Gross-Zagier, Kolyvagin and Zhang in the case $r_{2}(K / F)=[F: \mathbb{Q}]$ mentioned above. In spite of this, a plethora of conjectural constructions of points have been proposed so far in various settings beyond the classical one. These points are commonly called Stark-Heegner points, or Darmon points, as it was H. Darmon in Da2] who first introduced them.

Since then, several authors [Das1, Gr, LRV], DL, Ga1 have proposed variations of Darmon's theme, always giving rise to a recipe that allows one to attach, to a given ring class field extension $H / K$ satisfying the hypothesis of Conjecture 1.1, a point

$$
P_{H} \in E\left(H_{v}\right),
$$

rational over the completion $H_{v}$ of $H$ at some finite or archimedean place $v$ of $H$, which is conjectured to satisfy the following properties:

(SH1) $P_{H} \in E(H)$, 
(SH2) For any character $\chi: \operatorname{Gal}(H / K) \rightarrow \mathbb{C}^{\times}$, the point

$$
P_{\chi}:=\sum_{\sigma \in \operatorname{Gal}(H / K)} \chi(\sigma)^{-1} \sigma\left(P_{H}\right) \in E(H) \otimes_{\mathbb{Z}} \mathbb{C}
$$

is non-zero if and only if $L^{\prime}(E / K, \chi, 1) \neq 0$, and

(SH3) there is a reciprocity law describing the action of $\operatorname{Gal}(H / K)$ on $P_{H}$ in terms of ideal theory.

The main result of this paper is a new, computable construction of a supply of Darmon-like points in a setting that was not computationally accessible before. Before describing our contribution in more detail, we take the chance to report on the state of the art of Darmon points. Namely, explain which cases of Conjecture 1.1 are already covered by the union of those constructions, and which ones remain intractable.

Keep the above notation and the assumptions of Conjecture 1.1, and assume that $H$ is the narrow ring class field associated with some order in $K$. Then:

a) If $r_{1}(K / F)=0, r_{2}(K / F)=[F: \mathbb{Q}]$, then $\operatorname{assumption} \operatorname{sign}(E / K)=-1$ implies that (JL) holds, and Conjecture 1.1 holds thanks to $\mathrm{GZ}$, [Ko and Zh.

b) If $\sharp\left\{\wp \mid N^{-}\right\} \geqslant 1$, points $P_{H} \in E\left(H_{\wp}\right)$ have been constructed in Da2, Gr] and [LRV], for which conditions (SH1), (SH2) and (SH3) above have been conjectured.

Some theoretical evidence has been provided for them when $F=\mathbb{Q}$ in BD, GSS] and [LV].

Numerical evidence has been given in Da2 when $F=\mathbb{Q}$ and $N^{-}=1$.

c) If $r_{1}(K / F) \geqslant 1, r_{2}(K / F) \geqslant 1$ let us distinguish two possibilities:

c1) If $r_{2}(K / F)=1, K / F$ is called an almost totally real (ATR) quadratic extension and we let $v$ denote the unique archimedean place of $F$ which extends to a complex place of $K$. Then $H_{v}=\mathbb{C}$ for any place of $H$ above it, and points $P_{H} \in E\left(H_{v}\right)$ have been constructed in Da1, Ch. VIII], for which conditions (SH1), (SH2) and (SH3) above have been conjectured. These conjectures have been tested numerically in DL.

c2) J. Gartner has extended the idea of Darmon Da1, Ch. VIII] to any $K / F$ with $1 \leqslant r_{2}(K / F)<[F: \mathbb{Q}]$ : in this more general setting, he constructs points $P_{H} \in E\left(H_{v}\right)$ and again conjectures that (SH1), (SH2) and (SH3) hold true. His method does not appear to be amenable to explicit calculations and as a consequence no numerical evidence has been provided for these conjectures.

Note that a), b), c) cover all cases contemplated in Conjecture 1.1. Indeed, the only case not covered by b) arises when $\sharp\left\{\wp \mid N^{-}\right\}=0$, that is, all primes $\wp \mid N$ split or ramify in $K$. But then assumption $\operatorname{sign}(E / K)=-1$ implies that $r_{2}(K / F)$ is odd, hence $r_{2}(K / F) \geqslant 1$. Then a) and c) cover respectively the case in which $r_{1}(K / F)=0$ and $r_{1}(K / F)>0$.

The main contribution of this article is an explicitly computable construction of a supply of points $P_{M} \in E(\mathbb{C})$ in a setting which lies within c2), but which is completely different from the one proposed by Gartner. It only works under the following restrictive hypothesis:

- $F$ contains a field $F_{0}$ with $\left[F: F_{0}\right]=2$, 
- $E / F$ is $F$-isogenous to its Galois conjugate over $F_{0}$, and

- $M$ is an almost totally complex (ATC) quadratic extension 1 of $F$, that is to say, $r_{2}(M / F)=[F: \mathbb{Q}]-1$.

While this setting is obviously much less general than the one considered in Ga1, it enjoys the following features:

- Numerical approximations to the points $P_{M}$ are computable, as we illustrate with a fully detailed explicit example in $\$ 3.3$. Indeed, our construction relies on the computation of certain ATR cycles on Hilbert modular varieties. The algorithm of GM2 can be used to effectively compute such cycles on Hilbert modular varieties of the non-trivial level, and in combination with the explicit construction of ATC points that we give in $\$ 3.1$ (see especially Theorem 3.10) this gives an algorithm for computing ATC points.

- We prove that the points $P_{M}$ belong to $E(M)$ and that they are non-torsion if and only if $L^{\prime}(E / M, 1) \neq 0$ provided (SH1), (SH2) and (SH3) hold true for ATR extensions of $F_{0}$ : see Theorem 3.9 for the precise statement. This is worth remarking, as the conjectures for ATR extensions can be tested numerically in practice. See $\$ 2.4$ for a sketch of the algorithm, and DL, GM1 for explicit numerical examples.

The main source of inspiration for the construction presented here is the previous work DRZ of two of the authors with Henri Darmon, in which Heegner points on quotients of the modular curve $X_{1}(N)$ were used to manufacture ATR points on elliptic curves.

\section{QUADRATIC POINTS ON MODUlar ABELIAN VARIETIES}

The basis of the main construction of this note-which we explain in $\$ 3$-lies in Darmon's conjectural theory of points on modular elliptic curves over almost totally real (ATR) quadratic extensions of a totally real number field.

In a recent article, Darmon's theory has been generalized by Gartner Ga2 by considering quaternionic modular forms with respect to not necessarily split quaternion algebras over the base field. Although we do not exploit Gartner's construction here, our points do lie in a theoretical setting which is also covered by him, and therefore the natural question arises as to whether Gartner's points are equal to ours when both constructions are available. We address this issue in 3.2 . where we point out that Conjecture (BSD) implies that one is a non-zero multiple of the other; the difference between them is that ours are numerically accessible, and this stands as the main motivation of this article.

This section is devoted to reviewing the work of Darmon and Gartner, settling on the way the notation that shall be in force for the rest of this note. As Gartner's exposition Ga1], Ga2 is already an excellent account of the theory, here we choose to reword it in the classical language of Hilbert modular forms, under the simplifying hypothesis that the narrow class number of the base field $F_{0}$ is 1 .

In doing so, we take the opportunity to contribute to the theory with a few novel aspects. To name one, it will be convenient for our purposes to work with the natural, relatively straightforward extension of the theory to the setting of

\footnotetext{
${ }^{1}$ Observe the notational change here, which we introduce as being consistent with the notation we will use in 93 below, where two quadratic extensions of totally real fields will play a role in our construction: $M$ will denote an ATC extension of $F$ and $K$ and an ATR extension of $F_{0}$.
} 
eigenforms with not necessarily trivial nebentypus and whose eigenvalues generate a number field of arbitrarily large degree over $\mathbb{Q}$. This will lead us to a construction of rational points on higher-dimensional modular abelian varieties of $\mathrm{GL}_{2}$-type.

2.1. Quadratic extensions and $L$-functions. Let $F_{0} \subset \mathbb{R}$ be a totally real number field, together with a fixed embedding into the field of real numbers. Write $d=\left[F_{0}: \mathbb{Q}\right]$ for its degree over $\mathbb{Q}$ and let $R_{0} \subset F_{0}$ denote its ring of integers. In order to keep our notation simple, we assume that the narrow class number of $F_{0}$ is 1 .

Let $N$ be a squarefree integral ideal of $F_{0}$, and let $\psi$ be a Hecke character of conductor $N_{\psi}$ dividing $N$. Let $f_{0} \in S_{2}(N, \psi)$ be a normalized Hilbert eigenform of parallel weight 2 , level $N$ and nebentypus $\psi$. Let $E_{f_{0}}$ denote the number field generated by the eigenvalues of the Hecke operators acting on $f_{0}$, which we regard as embedded in the algebraic closure $\overline{\mathbb{Q}}$ of $\mathbb{Q}$ in the field $\mathbb{C}$ of complex numbers; for each $\sigma \in \operatorname{Hom}\left(E_{f_{0}}, \overline{\mathbb{Q}}\right)$, there exists a unique normalized eigenform ${ }^{\sigma} f_{0}$ whose family of eigenvalues is equal to the family of eigenvalues of $f_{0}$ conjugated by $\sigma$.

The following standard conjecture is a generalized form of the Eichler-Shimura philosophy.

Conjecture 2.1. There exists an abelian variety $A=A_{f_{0}} / F_{0}$ of dimension $g=$ $\left[E_{f_{0}}: \mathbb{Q}\right]$ and conductor $N^{g}$ such that $\mathbb{Q} \otimes \operatorname{End}_{F_{0}}(A) \simeq E_{f_{0}}$, and whose L-series factors as

$$
L(A, s)=\prod_{\sigma \in \operatorname{Hom}\left(E_{f_{0}}, \overline{\mathbb{Q}}\right)} L\left(^{\sigma} f_{0}, s\right) .
$$

Note that, if such an $A$ exists, it is well defined only up to isogenies.

Conjecture 2.1 is known to hold when (JL) is satisfied. When (JL) fails, it is not even known whether there exists a motive $M_{f_{0}}$ over $F$ whose $L$-function is (5) and one certainly does not expect the motive $h^{1}(A)$ to arise in the cohomology of any (quaternionic) Hilbert variety (cf. [BR] for more details). See [De] for the numerical verification of Conjecture 2.1 in several instances in which (JL) fails.

We shall assume for the remainder of this section that Conjecture2.1 holds true.

Let $K / F_{0}$ be a quadratic extension such that $r_{2}\left(K / F_{0}\right) \geqslant 1$. Label the set of embeddings of $F_{0}$ into the field $\mathbb{R}$ of real numbers as

$$
\left\{v_{1}, v_{2}, \ldots, v_{r}, v_{r+1}, \ldots, v_{d}: F_{0} \hookrightarrow \mathbb{R}\right\}, \quad 1 \leqslant r \leqslant d,
$$

in such a way that

- $v_{1}$ is the embedding fixed at the outset that we use to identify $F_{0}$ as a subfield of $\mathbb{R}$,

- each of the places $v_{2}, \ldots, v_{r}$ extends to a pair of real places of $K$, which by a slight abuse of notation we denote by $v_{j}$ and $v_{j}^{\prime}$ for each $j=2, \ldots, r$, and

- each of the places $v_{1}, v_{r+1}, \ldots, v_{d}$ extends to a complex place on $K$, that we still denote with the same letter; we use $v_{1}$ to regard $K$ as a subfield of $\mathbb{C}$.

Definition 2.2. If $r=1$, the set $\left\{v_{2}, \ldots, v_{r}\right\}$ is empty and $K / F_{0}$ is a CM-field extension.

If $r=2$ we call $K / F_{0}$ an almost totally complex (ATC) extension. To explain the terminology, observe that in this case $K$ has $[K: \mathbb{Q}]-2$ complex (non-real) embeddings, which is the maximum number of such embeddings a non-totally complex field of even degree can have. 
If $r=d$ we have $\left\{v_{1}, v_{r+1}, \ldots, v_{d}\right\}=\left\{v_{1}\right\}$ and we say that $K / F_{0}$ is almost totally real (ATR).

Letting $\varepsilon_{K}$ denote the quadratic Hecke character of $F_{0}$ associated with the extension $K / F_{0}$, the $L$-function of the base change of $A$ to $K$ is

$$
L(A / K, s)=L(A, s) \cdot L\left(A, \varepsilon_{K}, s\right)=\prod_{\sigma \in \operatorname{Hom}\left(E_{f_{0}}, \overline{\mathbb{Q}}\right)} L\left({ }^{\sigma} f_{0}, s\right) \cdot L\left({ }^{\sigma} f_{0}, \varepsilon_{K}, s\right) .
$$

It extends to an entire function on $\mathbb{C}$ and satisfies a functional equation relating the values at $s$ with $2-s$. Assume that the sign of the functional equation of $L\left(f_{0} / K, s\right)=L\left(f_{0}, s\right) \cdot L\left(f_{0}, \varepsilon_{K}, s\right)$ is -1 . This is equivalent to saying that the set

$$
\left\{v_{r+1}, \ldots, v_{d}\right\} \cup\{\wp \mid N, \wp \text { inert in } K\}
$$

has even cardinality.

Let $B$ be the (unique, up to isomorphism) quaternion algebra over $F_{0}$ whose set of places of ramification is equal to the set of places given in (6). In particular we have $B \otimes_{F_{0}, v_{j}} \mathbb{R} \simeq \mathrm{M}_{2}(\mathbb{R})$ for $j=1, \ldots, r$, and the choice of such isomorphisms gives rise to an embedding

$$
\left(v_{1}, \ldots, v_{r}\right): B^{\times} \hookrightarrow \mathrm{GL}_{2}(\mathbb{R}) \times\left(\stackrel{(r)}{\times} \times \mathrm{GL}_{2}(\mathbb{R}) \subset(B \otimes \mathbb{Q} \mathbb{R})^{\times} .\right.
$$

Let $N^{+}$be the product of primes in $F_{0}$ that divide $N$ and are split or ramified in $K$, and $N^{-}$be the product of primes that divide $N$ and remain inert in $K$. Choose an Eichler order $\mathcal{O}$ of level $N^{+}$in $B$ together with, for each prime $\wp \mid N^{+}$, isomorphisms $i_{\wp}: B \otimes F_{0, \wp} \simeq M_{2}\left(F_{0, \wp}\right)$ such that

$$
i_{\wp}(\mathcal{O})=\left\{\left(\begin{array}{ll}
a & b \\
c & d
\end{array}\right), \wp \mid c\right\} \subseteq \mathrm{M}_{2}\left(R_{0, \wp}\right) \text {. }
$$

Definition 2.3. Let $F_{0}^{+}$denote the subgroup of $F_{0}^{\times}$of totally positive elements and $B^{+}$be the subgroup of elements in $B^{\times}$whose reduced norm lies in $F_{0}^{+}$. Define the congruence subgroups

$$
\begin{gathered}
\Gamma_{0}=\Gamma_{0}^{N^{-}}\left(N^{+}\right)=\mathcal{O}^{\times} \cap B^{+} \text {and } \\
\Gamma_{1}=\Gamma_{1}^{N^{-}}\left(N^{+}\right)=\left\{\gamma \in \Gamma_{0}: i_{\wp}(\gamma) \equiv\left(\begin{array}{cc}
1 & \star \\
0 & 1
\end{array}\right) \quad(\bmod \wp) \text { for all } \wp \mid N^{+}\right\} \subset \Gamma_{0} .
\end{gathered}
$$

Through (7), $\Gamma_{1}$ acts on the cartesian product $\mathcal{H}^{r}=\mathcal{H}_{1} \times \ldots \times \mathcal{H}_{r}$ of $r$ copies of Poincaré's upper half-plane and we let $X_{\mathbb{C}}=\Gamma_{1} \backslash \mathcal{H}^{r}$ denote its quotient, which has a natural structure of analytic manifold with finitely many isolated singularities.

Definition 2.4. Let $F_{0}^{\text {gal }}$ denote the Galois closure of $F_{0}$ in $\mathbb{C}$ and let $G=$ $\operatorname{Gal}\left(F_{0}^{\text {gal }} / \mathbb{Q}\right)$. The reflex field of $B$ is the subfield $F_{0}^{\star}$ of $F_{0}^{\text {gal }}$ fixed by the subgroup of those $\sigma \in G$ such that $\sigma \cdot\left\{v_{1}, \ldots, v_{r}\right\}=\left\{v_{1}, \ldots, v_{r}\right\}$.

The cases one encounters most often in the literature arise when either $r=1$, where $F_{0}^{\star}=F_{0}$, or when $r=d$, in which case $F_{0}^{\star}=\mathbb{Q}$.

Let

$$
X=X_{1}^{N^{-}}\left(N^{+}\right) / F_{0}^{\star}
$$

denote Shimura's canonical model over $F_{0}^{\star}$ of $X_{\mathbb{C}}$, as introduced e.g. in [Mi1, §12]. If $\operatorname{Ram}(B) \neq \emptyset, X_{\mathbb{C}}$ is compact and $X$ is projective over $F_{0}^{\star}$, while if $\operatorname{Ram}(B)=\emptyset$, then $B=\mathrm{M}_{2}\left(F_{0}\right)$ and $X_{\mathbb{C}}$ may be compactified by adding a finite number of cusps. In both cases, the resulting projective variety admits a canonical desingularization. By an abuse of notation, we continue to denote $X$ as the smooth projective model over $F_{0}^{\star}$ that arises. 
2.2. Oda-Yoshida's conjecture. Let $\Sigma=\{ \pm 1\}^{r-1}$ and for each $\epsilon=\left(\epsilon_{2}, \ldots, \epsilon_{r}\right) \in$ $\Sigma$, let $\gamma_{\epsilon} \in \mathcal{O}^{\times}$be an element such that $v_{j}\left(n\left(\gamma_{\epsilon}\right)\right)=\operatorname{det}\left(v_{j}\left(\gamma_{\epsilon}\right)\right)>0$ if $j=1$ or $\epsilon_{j}=+1$, and $v_{j}\left(n\left(\gamma_{\epsilon}\right)\right)<0$ if $\epsilon_{j}=-1$. Such elements exist thanks to our running assumption that the narrow class number of $F_{0}$ is 1 . For $\tau_{j} \in \mathcal{H}_{j}$, set

$$
\tau_{j}^{\epsilon}=\left\{\begin{array}{l}
v_{j}\left(\gamma_{\epsilon}\right) \tau_{j} \text { if } j=1 \text { or } \epsilon_{j}=+1 \\
v_{j}\left(\gamma_{\epsilon}\right) \bar{\tau}_{j} \text { if } \epsilon_{j}=-1
\end{array}\right.
$$

Let $S_{2}\left(\Gamma_{1}\right)$ denote the set of weight two quaternionic cusp forms for $B$ with respect to the group $\Gamma_{1}=\Gamma_{1}^{N^{-}}\left(N^{+}\right)$. By the Jacquet-Langlands correspondence there exists a quaternionic newform $g_{0} \in S_{2}\left(\Gamma_{1}\right)$ with the same system of Hecke eigenvalues as $f_{0}$. In this way we can attach to $f_{0}$ the holomorphic $r$-form on $\mathcal{H}^{r}$

$$
\omega_{f_{0}}=(2 \pi i)^{r} g_{0}\left(\tau_{1}, \ldots, \tau_{r}\right) d \tau_{1} \ldots d \tau_{r}
$$

which is easily shown to be $\Gamma_{1}$-invariant (and to extend to a smooth form on the cusps, if $B=\mathrm{M}_{2}\left(F_{0}\right)$ ), giving rise to a regular differential $r$-form $\omega_{f_{0}} \in H^{0}\left(X_{\mathbb{C}}, \Omega^{r}\right)$.

Label the set $\operatorname{Hom}\left(E_{f_{0}}, \mathbb{C}\right)=\left\{\sigma_{1}, \ldots, \sigma_{g}\right\}$ as embeddings of $E_{f_{0}}$ into the field of complex numbers. The set $\left\{\sigma_{1}\left(\omega_{f_{0}}\right), \ldots, \sigma_{g}\left(\omega_{f_{0}}\right)\right\}:=\left\{\omega_{f_{0}^{\sigma_{1}}}, \ldots, \omega_{f_{0}^{\sigma_{g}}}\right\}$ is then a basis of the $f_{0}$-isotypical component of $H^{0}\left(X, \Omega^{r}\right)$.

Definition 2.5 ([Da1, (8.2)], Ga1, §2]). Let $d_{0}$ be a totally positive generator of the different ideal of $F_{0}$ and let $\beta: \Sigma \rightarrow\{ \pm 1\}$ be a character. The differential $r$-form $\omega_{f_{0}}^{\beta}$ on $X$ associated with $f_{0}$ and $\beta$ is

$$
\omega_{f_{0}}^{\beta}:=\left|d_{0}\right|^{-1 / 2}(2 \pi i)^{r} \sum_{\epsilon \in \Sigma} \beta(\epsilon) g_{0}\left(\tau_{1}^{\epsilon}, \ldots, \tau_{r}^{\epsilon}\right) d \tau_{1}^{\epsilon} \ldots d \tau_{r}^{\epsilon} .
$$

If $r=1$, note that the only choice for $\beta$ is the trivial one, and in this case one recovers the usual holomorphic 1 -form $\omega_{f_{0}}$ on the Shimura curve $X / F_{0}$. On the other hand, when $r>1$, the differential form $\omega_{f_{0}}^{\beta}$ is not holomorphic anymore for any choice of $\beta$, including the trivial one. Following Yoshida $[\mathrm{Yo}$, we define the following lattice.

Definition 2.6. The lattice of periods of $\omega_{f_{0}}^{\beta}$ is

$$
\Lambda_{f_{0}}^{\beta}=\left\{\left(\int_{\tilde{Z}} \sigma_{1}\left(\omega_{f_{0}}^{\beta}\right), \ldots, \int_{\tilde{Z}} \sigma_{g}\left(\omega_{f_{0}}^{\beta}\right)\right), \tilde{Z} \in H_{r}\left(X_{\mathbb{C}}, \mathbb{Z}\right)\right\} \subseteq \mathbb{C}^{g} .
$$

Under the running assumption that Conjecture 2.1 holds true, we can also introduce another lattice as follows. For each $j=1, \ldots, r$, let $A_{j}=A \times{ }_{F_{0}, v_{j}} \mathbb{C}$ denote the base change of $A$ to the field of complex numbers via the embedding $F_{0} \stackrel{v_{j}}{\hookrightarrow} \mathbb{R} \subset \mathbb{C}$. Since we identify $v_{1}$ with the identity embedding, $A_{1}$ is identified with $A$. Let $H_{1}\left(A_{j}, \mathbb{Z}\right)^{ \pm}$be the $\mathbb{Z}$-submodule of $H_{1}\left(A_{j}, \mathbb{Z}\right)$ on which complex conjugation acts as +1 (resp. -1). Since $E_{f_{0}} \simeq \mathbb{Q} \otimes \operatorname{End}_{F_{0}}(A)$, there is a natural action of $E_{f_{0}}$ on $H_{1}\left(A_{j}, \mathbb{Q}\right)^{ \pm}$and in fact the latter is a free module of rank 1 over the former.

Similarly, the space $H^{0}\left(A, \Omega^{1}\right)$ of global regular differential 1-forms on $A$ is an $F_{0}$-vector space of dimension $g$ equipped with an $F_{0}$-linear action of $E_{f_{0}}$ inherited from the isomorphism $E_{f_{0}} \simeq \mathbb{Q} \otimes \operatorname{End}_{F_{0}}(A)$.

Recall that $R_{0}$ stands for the ring of integers of $F_{0}$. Make the following choices:

- A regular differential $\omega_{A} \in H^{0}\left(A, \Omega^{1}\right)$ which extends to a smooth differential on the Néron model of $A$ over $R_{0}$ and generates $H^{0}\left(A, \Omega^{1}\right)$ as an $E_{f_{0}}$-module. 
- For each $j=1, \ldots, r$, generators $c_{j}^{+}, c_{j}^{-}$of $H_{1}\left(A_{j}, \mathbb{Q}\right)^{+}$and $H_{1}\left(A_{j}, \mathbb{Q}\right)^{-}$as $E_{f_{0}}$-modules.

Given these choices, define

$$
\begin{gathered}
\Omega_{j}^{+}=\int_{c_{j}^{+}} v_{j}\left(\omega_{A}\right) \in \mathbb{C}, \quad \Omega_{j}^{-}=\int_{c_{j}^{-}} v_{j}\left(\omega_{A}\right) \in \mathbb{C}, \text { for } j=1, \ldots, r \text { and } \\
\Omega_{\beta}=\Omega_{2}^{\beta_{2}(-1)} \cdot \ldots \cdot \Omega_{r}^{\beta_{r}(-1)} .
\end{gathered}
$$

Definition 2.7. Let $R_{f_{0}}$ denote the ring of integers of $E_{f_{0}}$ and define

$$
\Lambda_{0}^{\beta}:=\Omega_{\beta} \cdot\left(\mathbb{Z} \Omega_{1}^{+}+\mathbb{Z} \Omega_{1}^{-}\right) \subset \mathbb{C}, \quad \Lambda_{A}^{\beta}:=\Lambda_{0}^{\beta} \otimes_{\mathbb{Z}} R_{f_{0}} \subseteq \mathbb{C} \otimes_{\mathbb{Z}} E_{f_{0}} \simeq \mathbb{C}^{g} .
$$

Let us now analyze how these lattices depend on the above choices. Note that $\omega_{A}$ is well defined only up to multiplication by units $u \in R_{0}^{\times}$and non-zero endomorphisms $t \in E_{f_{0}}^{\times}$. If we replace $\omega_{A}$ by $u \cdot \omega_{A}$, we obtain

$$
\begin{gathered}
\Lambda_{0}^{\beta}\left(u \cdot \omega_{A}\right)=\left\langle\int_{c_{1}^{+}} v_{1}\left(u \cdot \omega_{A}\right) \cdot \prod_{j=2}^{r} \int_{c_{j}^{\beta_{j}(-1)}} v_{j}\left(u \cdot \omega_{A}\right), \int_{c_{1}^{-}} v_{1}\left(u \cdot \omega_{A}\right) \cdot \prod_{j=2}^{r} \int_{c_{j}^{\beta_{j}(-1)}} v_{j}\left(u \cdot \omega_{A}\right)\right\rangle \\
=\mathrm{N}_{F_{0} / \mathbb{Q}}(u) \Lambda_{0}^{\beta}\left(\omega_{A}\right)=\Lambda_{0}^{\beta}\left(\omega_{A}\right),
\end{gathered}
$$

because $\mathrm{N}_{F_{0} / \mathbb{Q}}(u)= \pm 1$, and thus also $\Lambda_{A}^{\beta}\left(u \cdot \omega_{A}\right)=\Lambda_{A}^{\beta}\left(\omega_{A}\right)$.

If instead we replace $\omega_{A}$ by $t \cdot \omega_{A}$ for some $t \in E_{f_{0}}^{\times}$, then

$$
\begin{gathered}
\Lambda_{A}^{\beta}\left(t \omega_{A}\right)=\left\{\Omega_{\beta} \int_{c_{1}^{+}} v_{1}\left(t^{*} \omega_{A}\right) \otimes s, \Omega_{\beta} \int_{c_{1}^{-}} v_{1}\left(t^{*} \omega_{A}\right) \otimes s, s \in R_{f_{0}}\right\} \\
=\left\{\Omega_{\beta} \int_{c_{1}^{+}} v_{1}\left(\omega_{A}\right) \otimes s t, \Omega_{\beta} \int_{c_{1}^{-}} v_{1}\left(\omega_{A}\right) \otimes s t, s \in R_{f_{0}}\right\}
\end{gathered}
$$

and therefore $\mathbb{Q} \otimes_{\mathbb{Z}} \Lambda_{A}^{\beta}\left(t \omega_{A}\right)=\mathbb{Q} \otimes_{\mathbb{Z}} \Lambda_{A}^{\beta}\left(\omega_{A}\right)$. We reach the same conclusion if we take different choices of homotopically equivalent paths $c_{j}^{+}$or $c_{j}^{-}$. Hence the $\mathbb{Q}$-submodule $\mathbb{Q} \otimes_{\mathbb{Z}} \Lambda_{A}^{\beta}$ of $\mathbb{C}^{g}$ is determined uniquely independently of the choices made.

Conjecture 2.8 (Oda $\mathrm{Od}$, Yoshida $[\mathrm{YO}]$ ). The lattices $\Lambda_{f_{0}}^{\beta}$ and $\Lambda_{A}^{\beta}$ are commensurable, that is to say,

$$
\mathbb{Q} \otimes_{\mathbb{Z}} \Lambda_{f_{0}}^{\beta}=\mathbb{Q} \otimes_{\mathbb{Z}} \Lambda_{A}^{\beta},
$$

and therefore there exists an isogeny of abelian varieties

$$
\eta_{\beta}: \mathbb{C}^{g} / \Lambda_{f_{0}}^{\beta} \stackrel{\sim}{\longrightarrow} \mathbb{C}^{g} / \Lambda_{A}^{\beta} \simeq A(\mathbb{C})=\mathbb{C}^{g} / \Lambda_{1},
$$

where the last isomorphism is given by multiplication by $\Omega_{\beta}^{-1}$.

Note that, consistently with Conjecture 2.1 the above Conjecture 2.8 only concerns the isogeny class of the abelian variety $A$.

Remark 2.9. If $r=1$ and (JL) holds, Conjecture 2.8 holds true: the abelian variety $A$ may be constructed explicitly as a constituent of the Jacobian of the Shimura curve $X$, and it follows from the very construction that the lattices $\Lambda_{f_{0}}^{\beta}$ and $\Lambda_{A}^{\beta}$ are commensurable. 
2.3. Darmon points. Let $\mathcal{Z}_{r-1}\left(X_{\mathbb{C}}\right)$ denote the set of null-homologous cycles of real dimension $r-1$ in $X_{\mathbb{C}}$. For each character $\beta$ as above, Conjecture 2.8 allows us to define the topological Abel-Jacobi map

$$
\begin{array}{ccc}
\mathrm{AJ}^{\beta}: \mathcal{Z}_{r-1}\left(X_{\mathbb{C}}\right) & \longrightarrow & A(\mathbb{C}), \\
T & \mapsto & \eta_{\beta}\left(\int_{\tilde{T}} \omega_{f_{0}}^{\beta}\right),
\end{array}
$$

where $\tilde{T} \in C_{r}\left(X_{\mathbb{C}}, \mathbb{Z}\right)$ is any $r$-dimensional chain satisfying $\partial \tilde{T}=T$. Observe that $\tilde{T}$ is determined up to elements in $H_{r}\left(X_{\mathbb{C}}, \mathbb{Z}\right)$, so that the quantity $\int_{\tilde{T}} \omega_{f_{0}}^{\beta} \in \mathbb{C} \otimes_{\mathbb{Z}} E_{f_{0}}$ is a well-defined element in $\mathbb{C}^{g} / \Lambda_{f_{0}}^{\beta}$ and $\mathrm{AJ}^{\beta}$ is thus a well-defined map.

Remark 2.10. If $r=1$ and (JL) holds, the map $\mathrm{AJ}^{\beta}$ is nothing else but the classical algebraic Abel-Jacobi map of curves $X_{\mathbb{C}} \longrightarrow A(\mathbb{C})$, which factors through the jacobian of $X_{\mathbb{C}}$. This was one of Darmon's motivations for extending the rule to the general case, though the reader must be warned that when $r>1$ the maps $\mathrm{AJ}^{\beta}$ are not algebraic.

Now let $c \subseteq R_{0}$ be an integral ideal of $F_{0}$ coprime to $N$ and let $R_{c}:=R_{0}+$ $c R_{K} \subseteq R_{K}$ be the order of conductor $c$ in the ring of integers of $K$. Let $\eta$ be the homomorphism

$$
\eta: \mathcal{O} \longrightarrow R_{0} / N^{+} R_{0}
$$

sending an element $x \in \mathcal{O}$ to the upper left-hand entry of its image in $\mathcal{O} \otimes_{R_{0}} R_{0, N^{+}} \simeq$ $\mathrm{M}_{2}\left(R_{0, N^{+}}\right)$, taken modulo $N^{+} R_{0, N^{+}}$.

Definition 2.11. Fix a factorization of ideals $N^{+} R_{K}=\mathfrak{N}^{+} \cdot \overline{\mathfrak{N}}^{+}$. An embedding of $R_{0}$-algebras $\varphi: R_{c} \hookrightarrow \mathcal{O}$ is said to be optimal if $\varphi\left(R_{c}\right)=\varphi(K) \cap \mathcal{O}$. We say that $\varphi$ is normalized (with respect to $\mathfrak{N}^{+}$) if it satisfies the following conditions:

(1) $\varphi$ acts on $u=\left(\tau_{1}, 1\right) \in \mathbb{C}^{2}$ as $\varphi(a)_{1} \cdot u=v_{1}(a) \cdot u$ for all $a \in R_{c}$, where $\varphi(a)_{1}$ denotes the image of $\varphi(a)$ in $B \otimes_{F_{0}, v_{1}} \mathbb{R}$.

(2) The kernel of $\eta \circ \varphi$ is equal to $\mathfrak{N}^{+}$.

We denote by $\mathcal{E}\left(R_{c}, \mathcal{O}\right)$ the set of normalized optimal embeddings.

Recall that $v_{1}$ extends to a complex place of $K$ and that $v_{2}, \ldots, v_{r}$ extend to real places. Given $\varphi \in \mathcal{E}\left(R_{c}, \mathcal{O}\right)$, the action of $K^{\times}$on $\mathbb{C}$ by fractional linear transformations via the composition of $\varphi$ and the isomorphism $\left(B \otimes_{F_{0}, v_{1}} \mathbb{R}\right)^{\times} \simeq \mathrm{GL}_{2}(\mathbb{R})$ has a unique fixed point $z_{1} \in \mathcal{H}_{1}$. For $j=2, \ldots, r$ it has two fixed points $\tau_{j}, \tau_{j}^{\prime} \in \mathbb{R}=\partial \mathcal{H}_{j}$ under the isomorphism $\left(B \otimes_{F_{0}, v_{j}} \mathbb{R}\right)^{\times} \simeq \mathrm{GL}_{2}(\mathbb{R})$. Let $\gamma_{j}$ be the geodesic path joining $\tau_{j}$ and $\tau_{j}^{\prime}$ in $\mathcal{H}_{j}$.

Definition 2.12. We denote by $T_{\varphi}$ the $(r-1)$-real-dimensional cycle in $X_{\mathbb{C}}$ given by the image of the region

$$
R_{\varphi}=\left\{z_{1}\right\} \times \gamma_{2} \times \cdots \times \gamma_{r} \subset \mathcal{H}_{1} \times \cdots \times \mathcal{H}_{r}
$$

under the natural projection map $\mathcal{H}^{r} \longrightarrow X_{\mathbb{C}}$.

Note that the stabilizer of $R_{\varphi}$ in $\Gamma_{1}$ is the subgroup $\Gamma_{\varphi}=\varphi(K) \cap \Gamma_{1}$ and therefore there is a natural homeomorphism $T_{\varphi} \simeq \Gamma_{\varphi} \backslash R_{\varphi}$. As an application of the Matsushima-Shimura Theorem [MS], it is easy to show (cf. Ga2, Proposition 4.3.1]) that the class of $T_{\varphi}$ has finite order in $H_{r-1}\left(X_{\mathbb{C}}, \mathbb{Z}\right)$. In particular, if $e$ denotes the order of $T_{\varphi}$, then $e T_{\varphi}$ is null-homologous. This allows the following definition. 
Definition 2.13. The Darmon point attached to $\varphi$ and $\beta$ is

$$
P_{\varphi}^{\beta}:=\frac{1}{e} \mathrm{AJ}^{\beta}\left(e T_{\varphi}\right) \in A_{1}(\mathbb{C}) .
$$

Darmon points are conjectured to be rational over certain number fields, with the Galois action given by an explicit reciprocity law. This is the content of Conjecture 2.14 Next we define the number fields and the actions involved in the conjecture.

Define

$$
U_{c}:=\hat{R}_{0}^{\times}\left(1+c \hat{R}_{K}\right) \subset \hat{K}^{\times}=\left(K \otimes_{\mathbb{Z}} \hat{\mathbb{Z}}\right)^{\times} .
$$

For every character $\beta$ of $\Sigma$, let $H_{c}^{\beta}$ denote the abelian extension of $K$ corresponding by class field theory to the open compact subgroup $K_{\infty}^{\beta} \times U_{c}$, where

$$
K_{\infty}^{\beta}:=\prod_{j=1, r+1, \ldots, d} \mathbb{C}^{\times} \times \prod_{\substack{j=2, \ldots, r \\ \beta_{j}(-1)=+1}} \mathbb{R}^{\times} \times \prod_{\substack{j=2, \ldots, r \\ \beta_{j}(-1)=-1}} \mathbb{R}_{>0} .
$$

Recall that $\psi$ denotes the Nebentypus of $f_{0}$. For $\varphi: R_{c} \hookrightarrow \mathcal{O}$ a normalized optimal embedding define

$$
\begin{aligned}
& U_{\varphi}^{+}=\operatorname{ker}\left(U_{c} \longrightarrow\left(R_{K} / \mathfrak{N}^{+} R_{K}\right)^{\times} \simeq\left(R_{0} / N^{+} R_{0}\right)^{\times} \stackrel{\psi}{\rightarrow} \mathbb{C}^{\times}\right), \\
& U_{\varphi}^{-}=\operatorname{ker}\left(U_{c} \longrightarrow\left(R_{K} / \overline{\mathfrak{N}}^{+} R_{K}\right)^{\times} \simeq\left(R_{0} / N^{+} R_{0}\right)^{\times} \stackrel{\psi}{\rightarrow} \mathbb{C}^{\times}\right) .
\end{aligned}
$$

Denote by $L_{\varphi}^{\beta} / H_{c}^{\beta}$ (resp. $L_{\varphi}^{\prime \beta} / H_{c}^{\beta}$ ) the abelian extension of the ring class field of conductor $c$ associated to $K_{\infty}^{\beta} \times U_{\varphi}^{+}$(resp. $\left.K_{\infty}^{\beta} \times U_{\varphi}^{-}\right)$. Also let $U_{\varphi}=U_{\varphi}^{+} \cap U_{\varphi}^{-}$and let $H_{\varphi}^{\beta} / H_{c}^{\beta}$ be the extension associated to $K_{\infty}^{\beta} \times U_{\varphi} \subset \mathbb{A}_{K}^{\times}$.

Observe that we can extend $\psi$ to a character on $\mathcal{O}^{\times}$by composing with $\eta$. Then we define

$$
\Gamma_{1} \subseteq \Gamma_{\psi}:=\Gamma_{\psi}^{N^{-}}\left(N^{+}\right):=\left\{\gamma \in \Gamma_{0}: \psi(\eta \gamma)=1\right\} \subseteq \Gamma_{0} .
$$

The group $\Gamma_{0}$ acts on $\mathcal{E}\left(R_{c}, \mathcal{O}\right)$ by conjugation, and we denote by $\mathcal{E}\left(R_{c}, \mathcal{O}\right) / \Gamma_{0}$ the set of conjugacy classes. Any element $W_{\varepsilon} \in \Gamma_{0} \backslash \Gamma_{\psi}$ defines an involution on $\mathcal{E}\left(R_{c}, \mathcal{O}\right) / \Gamma_{0}$ which interchanges the preimages of the natural projection $\mathcal{E}\left(R_{c}, \mathcal{O}\right) / \Gamma_{\psi}$ $\rightarrow \mathcal{E}\left(R_{c}, \mathcal{O}\right) / \Gamma_{0}$. In addition to $W_{\varepsilon}$, there is also an Atkin-Lehner involution acting naturally on the set of embeddings, although it does not preserve the normalization. To be more precise, let $\omega_{N}$ be an element in $B$ such that

- for every $\wp \mid N^{-}, \omega_{N}$ generates the single two-sided ideal of $\mathcal{O} \otimes R_{0, \wp}$ of norm $\wp$, and

- for every $\wp \mid N^{+}, \iota_{\wp}\left(\omega_{N}\right)=\left(\begin{array}{cc}0 & -1 \\ \pi_{\wp} & 0\end{array}\right)$, where $\pi_{\wp}$ is any uniformizer in $R_{0, \wp}$.

Let us denote by $\overline{\mathcal{E}}\left(R_{c}, \mathcal{O}\right)$ the set of optimal embeddings normalized with respect to $\overline{\mathfrak{N}}^{+}$. Then the map $\varphi \mapsto W_{N}(\varphi):=\omega_{N} \varphi \omega_{N}^{-1}$ is a bijection between $\mathcal{E}\left(R_{c}, \mathcal{O}\right)$ and $\overline{\mathcal{E}}\left(R_{c}, \mathcal{O}\right)$. From now on denote by $W_{N}\left(P_{\varphi}^{\beta}\right)$ the point $P_{W_{N}(\varphi)}^{\beta}$.

Finally, there is also a natural action of $\hat{K}^{\times}$on $\mathcal{E}\left(R_{c}, \mathcal{O}\right)$, which works as follows. Pick a finite idèle $x \in \hat{K}^{\times}$and an embedding $\varphi$ in $\mathcal{E}\left(R_{c}, \mathcal{O}\right)$. Since the class number of $\mathcal{O}$ is $h(\mathcal{O})=h\left(F_{0}^{\star}\right)=1$ by [Vi, Cor. 5.7 bis], the fractional ideal $I_{x}=\varphi(x) \hat{\mathcal{O}} \cap B$ is principal, generated by some $\gamma_{x} \in B^{\times}$with $n\left(\gamma_{x}\right) \in F_{0}^{+}$. Moreover, we can choose $\gamma_{x}$ such that $a_{x}=\varphi\left(x_{\mathfrak{N}^{+}} x_{\overline{\mathfrak{N}}^{+}}\right)^{-1} \cdot \gamma_{x}$ lies in the kernel of $\psi \eta$. (Indeed, note that, locally at the primes $\wp \mid N^{+}$, we have $\varphi\left(x_{\mathfrak{N}^{+}} x_{\overline{\mathfrak{N}}^{+}}\right)^{-1} I_{x, \wp}=\mathcal{O}_{\wp}$ and thus $a_{x}$ belongs to $\mathcal{O}_{\wp}^{\times}$. It hence makes sense to consider its image under $\psi \eta$. We can 
assume $\gamma_{x}$ is as claimed by replacing it with a suitable unit in $\mathcal{O}^{\times}$.) We define $x \star \varphi:=\gamma_{x}^{-1} \circ \varphi^{\circ} \gamma_{x}$. Observe that $U_{\varphi}^{+}$acts trivially on $\mathcal{E}\left(R_{c}, \mathcal{O}\right)$.

For $y \in K_{\infty}^{\times}$and a character $\beta: \Sigma \rightarrow\{ \pm 1\}$, set

$$
\beta(y)=\prod_{j=2}^{r} \beta\left(\operatorname{sign}\left(\prod_{w \mid v_{j}} y_{w}\right)\right) .
$$

The following statement collects, in a precise form, the conjectures (SH1), (SH2), (SH3) that were somewhat vaguely formulated in the introduction for Darmon points over abelian extensions of $K$.

Conjecture 2.14. (1) If $\varphi \in \mathcal{E}\left(R_{c}, \mathcal{O}\right)$, then $P_{\varphi}^{\beta} \in A_{1}\left(L_{\varphi}^{\beta}\right)$.

(2) For any character $\chi: \operatorname{Gal}\left(L_{\varphi}^{\beta} / K\right) \rightarrow \mathbb{C}^{\times}$, the point

$$
P_{\chi}:=\sum_{\sigma \in \operatorname{Gal}\left(L_{\varphi}^{\beta} / K\right)} \chi(\sigma)^{-1} \sigma\left(P_{\varphi}^{\beta}\right) \in A_{1}\left(L_{\varphi}^{\beta}\right) \otimes_{\mathbb{Z}} \mathbb{C}
$$

is non-zero if and only if $L^{\prime}\left(f_{0} / K, \chi, 1\right) \neq 0$.

(3) For any $a=\left(a_{\infty}, a_{f}\right) \in \mathbb{A}_{K}^{\times}$we have that $\operatorname{rec}(a) P_{\varphi}^{\beta}=\beta\left(a_{\infty}\right) P_{a_{f} \star \varphi}$. In addition, for any $\tau \in \operatorname{Gal}\left(H_{\varphi}^{\beta} / F_{0}\right)$ whose restriction to $K$ is not trivial, there exists an element $\sigma \in \operatorname{Gal}\left(H_{\varphi}^{\beta} / K\right)$ such that

$$
\tau\left(P_{\varphi}^{\beta}\right)=W_{N}\left(\sigma\left(P_{\varphi}^{\beta}\right)\right)\left(\bmod A_{1}\left(H_{\varphi}^{\beta}\right)_{\text {tors }}\right) .
$$

Here rec $: \mathbb{A}_{K}^{\times} \longrightarrow \operatorname{Gal}\left(K^{\mathrm{ab}} / K\right)$ is Artin's reciprocity map, normalized so that uniformizers correspond to geometric Frobenius elements. Note that the three statements of Conjecture 2.14 are the translation to the current context of (SH1), (SH2), (SH3) given in the introduction.

2.4. Darmon-Logan's algorithm for the computation of ATR points. One naturally wonders whether Darmon points, as introduced in Definition 2.13, can be computed effectively in explicit examples. A positive answer would allow us to test Conjecture 2.14 numerically, leading to an explicit construction of rational points on elliptic curves over number fields which were not previously accessible.

However, the image of $T_{\varphi}$ under the Abel-Jacobi map AJ ${ }^{\beta}$ of (8) can only be computed provided we are able to write an explicit candidate for a region $\tilde{T}_{\varphi}$ having $T_{\varphi}$ as a boundary, and we can integrate it against the differential form $\omega_{f_{0}}^{\beta}$. The latter only seems possible when there is a natural, explicit description of $\omega_{f_{0}}^{\beta}$ available. This is precisely the case when the following Gross-Zagier assumption holds:

Assumption 2.15. $r=d$ and all the primes dividing $N$ are split or ramified in $K / F_{0}$.

Indeed, when this is the case we have that $K / F_{0}$ is an ATR extension, $B \simeq$ $\mathrm{M}_{2}\left(F_{0}\right), N=N^{+}, N^{-}=1$ and $X$ is a $d$-dimensional Hilbert modular variety over $F_{0}^{\star}=\mathbb{Q}$. In addition, and most importantly, the form $\omega_{f_{0}}^{\beta}$ admits a natural Fourier expansion around the cusp at infinity, and there exist algorithms which allow its computation up to a given precision; cf. e.g. [DV].

If Assumption 2.15] does not hold, we are at a loss to compute numerical approximations to the points $P_{\varphi}^{\beta}$. We impose Assumption 2.15 for the remainder of this section, that we devote to sketching Darmon-Logan's algorithm for computing an 
explicit chain $\tilde{T}_{\varphi}$ whose boundary is $T_{\varphi}$. We adapt it to our slightly more general setting in which $\left[E_{f_{0}}: \mathbb{Q}\right] \geqslant 1$, so that we can also make use of it later. To simplify the exposition, and since this is the case encountered in the numerical example described in 33.3 , let us also assume that $\left[F_{0}: \mathbb{Q}\right]=2$.

The key point in Darmon-Logan's approach is the definition of certain 3-limit integrals of $\omega_{f_{0}}^{\beta}$, allowed by the following interpretation of the homology groups of $X$. Let $\Gamma$ denote the quotient of $\Gamma_{\psi}$ by the normal closure of the subgroup generated by the elliptic and parabolic elements. Let $I_{\Gamma}$ be the augmentation ideal, which sits in the exact sequence

$$
0 \longrightarrow I_{\Gamma} \longrightarrow \mathbb{Z}[\Gamma] \longrightarrow \mathbb{Z} \longrightarrow 0
$$

For a $\Gamma$-module $M$ we denote by $M_{\Gamma}=M / I_{\Gamma} M$ its ring of $\Gamma$-coinvariants. Tensoring the above sequence by $I_{\Gamma}$ and taking the group homology exact sequence we obtain

$$
0 \longrightarrow H_{1}\left(\Gamma, I_{\Gamma}\right) \longrightarrow\left(I_{\Gamma} \otimes_{\mathbb{Z}} I_{\Gamma}\right)_{\Gamma} \stackrel{\partial}{\longrightarrow}\left(Z[\Gamma] \otimes_{\mathbb{Z}} I_{\Gamma}\right)_{\Gamma} \longrightarrow\left(I_{\Gamma}\right)_{\Gamma} \longrightarrow 0
$$

where $\partial$ is the natural map induced by the inclusion $I_{\Gamma} \subset Z[\Gamma]$. There are canonical isomorphisms $\left(I_{\Gamma}\right)_{\Gamma} \simeq H_{1}(\Gamma, \mathbb{Z})$ and $H_{1}\left(\Gamma, I_{\Gamma}\right) \simeq H_{2}(\Gamma, \mathbb{Z})$. Therefore, in view of the natural isomorphisms $H_{1}(\Gamma, \mathbb{Z}) \simeq H_{1}(X, \mathbb{Z})$ and $H_{2}(\Gamma, \mathbb{Z}) \simeq H_{2}(X, \mathbb{Z})$ one can identify (10) with the exact sequence

$$
0 \longrightarrow Z_{2}(X, \mathbb{Z}) \longrightarrow C_{2}(X, \mathbb{Z}) \stackrel{\partial}{\longrightarrow} Z_{1}(X, \mathbb{Z}) \longrightarrow H_{1}(X, \mathbb{Z}) \longrightarrow 0,
$$

where $\partial$ is the topological boundary map.

Recall that integrals of $\omega_{f_{0}}^{\beta}$ satisfy the following invariance property:

$$
\int_{x}^{y} \int_{z}^{t} \omega_{f_{0}}^{\beta}=\int_{\gamma x}^{\gamma y} \int_{\gamma z}^{\gamma t} \omega_{f_{0}}^{\beta}, \quad \text { for all } \gamma \in \Gamma \text { and } x, y, z, t \in \mathcal{H} .
$$

We remark that in this expression $\gamma$ is acting on the outer limits (resp. inner limits) of the integral through $v_{1}$ (resp. $v_{2}$ ). By choosing base points $z_{1} \in \mathcal{H}_{1}$ and $z_{2} \in \mathcal{H}_{2}$ one then obtains a group homomorphism

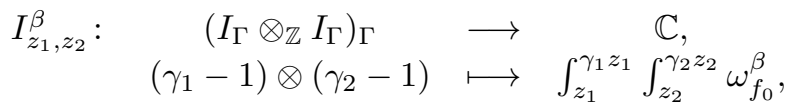

which can be identified with the map

$$
\begin{gathered}
C_{2}(X, \mathbb{Z}) \quad \longrightarrow \quad \mathbb{C}, \\
T \\
\qquad \int_{T} \omega_{f_{0}}^{\beta} .
\end{gathered}
$$

Observe that the identification $H_{1}\left(\Gamma, I_{\Gamma}\right) \simeq Z_{2}(X, \mathbb{Z})$ then yields an explicit description of the lattice $\Lambda_{f_{0}}^{\beta}$; indeed $\Lambda_{f_{0}}^{\beta} \simeq I_{z_{1}, z_{2}}^{\beta}\left(H_{1}\left(\Gamma, I_{\Gamma}\right)\right)$.

Suppose now that $1 \otimes\left(\gamma_{2}-1\right) \in \mathbb{Z}[\Gamma] \otimes I_{\Gamma}$ is such that $e\left(1 \otimes\left(\gamma_{2}-1\right)\right)$ lies in the image of $\partial$ for some integer $e$. That would correspond in (11) to a cycle $T$ such that $e T$ is null homologous. Following DL one defines

$$
\int^{z_{1}} \int_{z_{2}}^{\gamma_{2} z_{2}} \omega_{f_{0}}^{\beta}:=\frac{1}{e} I_{z_{1}, z_{2}}^{\beta}\left(\partial^{-1}\left(e \cdot\left(1 \otimes\left(\gamma_{2}-1\right)\right)\right)\right) \in \mathbb{C} / \tilde{\Lambda}_{f_{0}}^{\beta},
$$

where $\tilde{\Lambda}_{f_{0}}^{\beta}=\frac{1}{e} \Lambda_{f_{0}}^{\beta}$. This is indeed a well-defined quantity in $\mathbb{C} / \tilde{\Lambda}_{f_{0}}^{\beta}$, because any two preimages of $e\left(1 \otimes\left(\gamma_{1}-1\right)\right)$ by $\partial$ differ by an element of $Z_{2}(X, \mathbb{Z})$. It is sometimes convenient to use expressions such as $\int^{x} \int_{y}^{z} \omega_{f_{0}}^{\beta}$, but we warn the reader that they only make sense if $z=\gamma y$ for some $\gamma \in \Gamma$ and $e \cdot(1 \otimes \gamma) \in \operatorname{im}(\partial)$ for some $e$. It 
follows from the definitions that the 3-limit integrals of (12) enjoy the following properties:

$$
\begin{gathered}
\int^{x} \int_{y}^{z} \omega_{f_{0}}^{\beta}=\int^{\gamma x} \int_{\gamma y}^{\gamma z} \omega_{f_{0}}^{\beta} \quad \text { for all } \gamma \in \Gamma, \\
\int^{x} \int_{y}^{z} \omega_{f_{0}}^{\beta}=\int^{x} \int_{y}^{t} \omega_{f_{0}}^{\beta}+\int^{x} \int_{t}^{z} \omega_{f_{0}}^{\beta}, \\
\int^{y} \int_{t}^{z} \omega_{f_{0}}^{\beta}-\int^{x} \int_{t}^{z} \omega_{f_{0}}^{\beta}=\int_{x}^{y} \int_{t}^{z} \omega_{f_{0}}^{\beta} .
\end{gathered}
$$

Now let $K / F_{0}$ be a quadratic ATR extension and let $\varphi: R_{c} \hookrightarrow \mathcal{O}$ be a normalized optimal embedding of conductor $c$. Denote by $z_{1}$ the unique fixed point of $K$ acting on $\mathcal{H}_{1}$ through $v_{1}$. The stabilizer $\Gamma_{\varphi}$ of $z_{1}$ in $\Gamma$ is an abelian group or rank 1 (cf. [DL, Proposition 1.4]). Let $\gamma_{\varphi}$ be one of its generators. Let $z_{2}, z_{2}^{\prime} \in \partial \mathcal{H}_{2}$ denote the two fixed points of $K$ acting through $v_{2}$. Then we have that

$$
\int_{\tilde{T}_{\varphi}} \omega_{f_{0}}^{\beta}=\int^{z_{1}} \int_{z_{2}}^{z_{2}^{\prime}} \omega_{f_{0}}^{\beta}=\int^{z_{1}} \int_{z_{2}}^{\gamma_{\varphi} z_{2}} \omega_{f_{0}}^{\beta} .
$$

Using properties (13), (14) and (15) it is easy to check that the last integral does not depend on $z_{2}$. Therefore, we see that

$$
\int_{\tilde{T}_{\varphi}} \omega_{f_{0}}^{\beta}=\int^{z_{1}} \int_{x}^{\gamma_{\varphi} x} \omega_{f_{0}}^{\beta}
$$

for any $x \in \mathcal{H}_{2} \cup \mathbb{P}^{1}\left(F_{0}\right)$. If $N=1$ an algorithm for computing 3-limit integrals as the one in (16) is given in [DL, $\S 4]$ by means of the continued fractions trick. This has been generalized for arbitrary level $N$ in [GM2], replacing continued fractions by certain elementary matrix decompositions.

\section{Almost totally complex points}

This section is devoted to the main construction of the article. Recall that for a field extension $F / F_{0}$, an elliptic curve $E / F$ is said to be an elliptic $F_{0}$-curve if it is isogenous over $F$ to all of its $\operatorname{Gal}\left(F / F_{0}\right)$-conjugates. We give an explicitly computable construction of points on certain elliptic $F_{0}$-curves. By granting the conjectures of $\S 2$ over ATR extensions, these points are shown to be rational over ATC fields.

The construction of ATC points on $F_{0}$-curves is given in 33.1 . In 33.2 we compare our ATC points with Gartner's Darmon points, and conjecture a precise relation between them. Finally, in $\$ 3.3$ we carry out an explicit calculation of such an ATC point for a particular elliptic curve. At the same time as giving the details of how explicit computations can be handled, we numerically verify that the obtained point appears to satisfy the conjectures of $\$ 2$, which provides evidence for their validity.

3.1. Construction of ATC points. Let $F_{0}$ be a totally real number field of narrow class number 1 and degree $r$. We denote by $v_{1}, \ldots, v_{r}$ the embeddings of $F_{0}$ into $\mathbb{R}$, and we regard $F_{0}$ as a subfield of $\mathbb{C}$ via $v_{1}$. We will also regard all extensions of $F_{0}$ as embedded in $\mathbb{C}$ via a fixed extension of $v_{1}$ to $\bar{F}_{0}$, which we denote by $v_{1}$ as well. Let $F / F_{0}$ be a totally real quadratic extension and let $E / F$ be an elliptic $F_{0}$-curve without complex multiplication and of conductor $N_{E}$, which we assume to be squarefree and relatively prime to $\operatorname{disc}\left(F / F_{0}\right)$. Because $E$ is isogenous to its 
conjugate, $N_{E}$ is an integral ideal of $F$ of the form $N_{E}=N_{0} \cdot R_{F}$ for some ideal $N_{0}$ of $F_{0}$.

We denote by $A=\operatorname{Res}_{F / F_{0}} E$ the variety over $F_{0}$ obtained by restriction of scalars. The algebra $\mathbb{Q} \otimes \operatorname{End}_{F_{0}}(A)$ of endomorphisms of $A$ is then quadratic over $\mathbb{Q}$. The most interesting case for the arithmetic applications that we have in mind arises when $\mathbb{Q} \otimes \operatorname{End}_{F_{0}}(A)$ is a quadratic imaginary field, which we assume for the remainder of the article. In addition, we must assume that $A$ is modular:

Assumption 3.1. There exists a normalized Hilbert modular form $f_{0}$ over $F_{0}$ of parallel weight 2 such that (5) holds; that is to say,

$$
L(A, s)=\prod_{\sigma \in \operatorname{Hom}\left(E_{f_{0}}, \overline{\mathbb{Q}}\right)} L\left({ }^{\sigma} f_{0}, s\right) .
$$

In other words, $A=A_{f_{0}}$ up to $F_{0}$-isogenies. Observe also that, since $E$ is an $F_{0}$-curve, $A$ is isogenous over $F$ to $E^{2}$.

For any ideal $\mathfrak{m}$ of $F_{0}$, denote by $a_{\mathfrak{m}}$ the Fourier coefficient of $f_{0}$ corresponding to $\mathfrak{m}$.

Lemma 3.2. The level of $f_{0}$ is $N=N_{0} \cdot \operatorname{disc}\left(F / F_{0}\right)$. The nebentypus $\psi$ of $f_{0}$ is the quadratic character associated by class field theory to the extension $F / F_{0}$.

Proof. From the Eichler-Shimura construction (cf. Conjecture2.1) $A$ has conductor $N^{2}$. On the other hand, since $A$ is the restriction of scalars of $E$, its conductor is given by the formula

$$
\operatorname{Nm}_{F / F_{0}}\left(N_{E}\right) \operatorname{disc}\left(F / F_{0}\right)^{2}=N_{0}^{2} \operatorname{disc}\left(F / F_{0}\right)^{2},
$$

from which the first assertion follows. Let us now prove the second statement. Denote by $F_{\psi}$ the field cut by the kernel of $\psi$. Let $G=\operatorname{Gal}\left(\overline{\mathbb{Q}} / F_{0}\right), H=\operatorname{Gal}(\overline{\mathbb{Q}} / F)$ and $H_{\psi}=\operatorname{Gal}\left(\overline{\mathbb{Q}} / F_{\psi}\right)$. It is enough to show that $H=H_{\psi}$ (the fact that $\psi$ is quadratic follows from this because $\left.\left[F: F_{0}\right]=2\right)$. Let $\ell$ be a prime number that splits in $E_{f_{0}}$, say as $\ell=\lambda \lambda^{\prime}$, and denote by $V_{\ell}=T_{\ell}(A) \otimes_{\mathbb{Z}_{\ell}} \mathbb{Q}_{\ell}$ the $\ell$-adic Tate module of $A$. There is an isomorphism of $\mathbb{Q}_{\ell}[G]$-modules $V_{\ell}=V_{\lambda} \times V_{\lambda^{\prime}}$, where $V_{\lambda}=E_{\lambda} \otimes_{E \otimes \mathbb{Q}_{\ell}} V_{\ell}$ and $V_{\lambda^{\prime}}=E_{\lambda^{\prime}} \otimes_{E \otimes \mathbb{Q}_{\ell}} V_{\ell}$.

Denote by $\rho_{\lambda}$ and $\rho_{\lambda}^{\prime}$ the representations of $G$ afforded by $V_{\lambda}$ and $V_{\lambda^{\prime}}$ respectively, which are irreducible because $E$ is not CM. Since $A$ is the variety attached to $f_{0}$ by the Eichler-Shimura construction, and relabeling $\lambda$ and $\lambda^{\prime}$ if necessary, we can suppose that

$$
\operatorname{Tr}\left(\rho_{\lambda}\left(\text { Frob }_{\mathfrak{p}}\right)\right)=a_{\mathfrak{p}} \quad \text { and } \quad \operatorname{Tr}\left(\rho_{\lambda^{\prime}}\left(\text { Frob }_{\mathfrak{p}}\right)\right)=\bar{a}_{\mathfrak{p}}, \quad \text { for all primes } \mathfrak{p} \nmid N,
$$

where the bar denotes complex conjugation. By [Sh, Theorem 2.5] the nebentypus $\psi$ is characterized by the fact that $a_{\mathfrak{p}}=\bar{a}_{\mathfrak{p}} \psi(\mathfrak{p})$ for primes $\mathfrak{p} \nmid N$. Therefore $V_{\lambda}$ and $V_{\lambda^{\prime}}$ are isomorphic as $\mathbb{Q}_{\ell}\left[H_{\psi}\right]$ representations, so that $\operatorname{End}_{\mathbb{Q}_{\ell}\left[H_{\psi}\right]} V_{\ell} \simeq \mathrm{M}_{2}\left(\mathbb{Q}_{\ell}\right)$. Moreover, $H_{\psi}$ is the largest subgroup of $G$ for which this is true. On the other hand, we have that $\operatorname{End}_{F}(A) \otimes \mathbb{Q} \simeq \operatorname{End}_{F}\left(E^{2}\right) \otimes \mathbb{Q} \simeq \mathrm{M}_{2}(\mathbb{Q})$. By the case of Tate's Conjecture proven by Faltings, this implies that $\operatorname{End}_{\mathbb{Q}_{\ell}[H]} V_{\ell} \simeq \operatorname{End}_{F}^{0}(A) \otimes_{\mathbb{Q}} \mathbb{Q}_{\ell} \simeq$ $\mathrm{M}_{2}\left(\mathbb{Q}_{\ell}\right)$, from which we deduce that necessarily $H=H_{\psi}$.

Observe that, by the conductor-discriminant formula, the conductor $N_{\psi}$ of $\psi$ is equal to $\operatorname{disc}\left(F / F_{0}\right)$. For simplicity we assume from now on that $\operatorname{disc}\left(F / F_{0}\right)$ is not divisible by any prime above 2 , and thus $N=N_{0} N_{\psi}$ is squarefree. 
Let $M=F(\sqrt{\alpha})$ be a quadratic ATC extension of $F$ with $\operatorname{disc}(M / F)$ prime to $N_{E}$. Recall that ATC stands for almost totally complex, and it means in this case that $M$ has exactly two real places. We suppose that $M$ is real under the place $v_{1}$. We aim to give an explicitly computable construction of points in $E(M)$, by making use of the conjectural constructions of $₫ 2$,

Write $\operatorname{Gal}\left(F / F_{0}\right)=\{1, \tau\}$ and let $M^{\prime}=F\left(\sqrt{\alpha^{\tau}}\right)$. Clearly $M$ is not Galois over $F_{0}$, and its Galois closure $\mathcal{M}$ is the composition of $M$ and $M^{\prime}$. It is easily seen that $\operatorname{Gal}\left(\mathcal{M} / F_{0}\right) \simeq D_{8}$, the dihedral group of order 8 . The field $K=F_{0}\left(\sqrt{\alpha \alpha^{\tau}}\right)$ is contained in $\mathcal{M}$, and there exist quadratic extensions $L / K, L / K^{\prime}$ and $K^{\prime} / F_{0}$ such that the diagram of subfields of $\mathcal{M} / F_{0}$ is given by

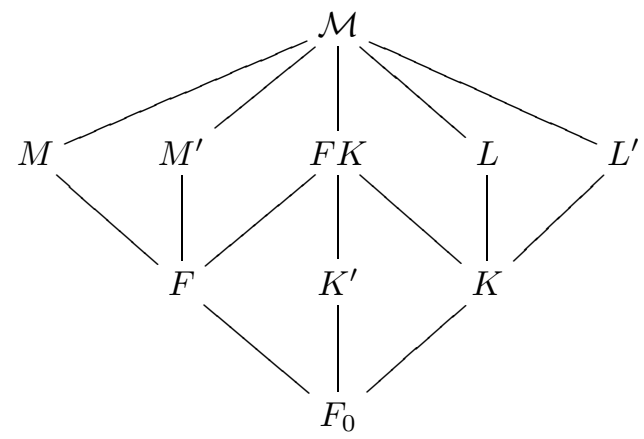

Our construction relies on the fact that $K$ is ATR. Indeed, we have the following lemma.

Lemma 3.3. The field $K$ is ATR and it is complex under $v_{1}$. The fields $L$ and $L^{\prime}$ are totally imaginary.

Proof. The first assertion follows immediately from the definitions. The property about $L$ comes from the fact that it can be identified with $K\left(\sqrt{\alpha}+\sqrt{\alpha^{\tau}}\right)=$ $K\left(\sqrt{\alpha+\alpha^{\tau}+2 \sqrt{\alpha \alpha^{\tau}}}\right)$, and similarly for $L^{\prime}$. Since $M$ is ATC, under a complex embedding of $L$ the image of either $\sqrt{\alpha}$ or $\sqrt{\alpha^{\tau}}$ does not lie in $\mathbb{R}$.

Let $\chi_{M}, \chi_{M^{\prime}}: \mathbb{A}_{F}^{\times} \rightarrow\{ \pm 1\}$ and $\chi_{L}, \chi_{L^{\prime}}: \mathbb{A}_{K}^{\times} \rightarrow\{ \pm 1\}$ denote the quadratic Hecke characters corresponding to the fields $M, M^{\prime}, L$ and $L^{\prime}$. Similarly, let $\varepsilon_{F}, \varepsilon_{K}: \mathbb{A}_{F_{0}}^{\times} \rightarrow\{ \pm 1\}$ be the ones corresponding to $F$ and $K$. Recall that $\varepsilon_{F}=\psi$ by Lemma 3.2 .

Lemma 3.4. (1) $\chi_{L} \chi_{L^{\prime}}=\psi \circ \mathrm{Nm}_{K / F_{0}}$.

(2) The restriction of $\chi_{L}$ to $\mathbb{A}_{F_{0}}^{\times}$is equal to $\psi$.

(3) We have that $\operatorname{Ind}_{F}^{F_{0}} \chi_{M} \simeq \operatorname{Ind}_{F}^{F_{0}} \chi_{M^{\prime}} \simeq \operatorname{Ind}_{K}^{F_{0}} \chi_{L} \simeq \operatorname{Ind}_{F}^{F_{0}} \chi_{L^{\prime}}$ are isomorphic as representations of $\operatorname{Gal}\left(\mathcal{M} / F_{0}\right)$.

Proof. Assertion (1) follows from the fact that $\chi_{L} \chi_{L^{\prime}}$ is the quadratic character associated with the extension $F K / K$, which is $\psi \circ \mathrm{Nm}_{K / F_{0}}$. If we let $\sigma$ denote the generator of $\operatorname{Gal}\left(K / F_{0}\right)$, then we have that $\chi_{L}\left(x^{\sigma}\right)=\chi_{L^{\prime}}(x)$. Then from (1) we see that $\chi_{L}$ restricted to $\mathrm{Nm}_{K / F_{0}} \mathbb{A}_{K}^{\times}$is equal to $\psi$. Then by class field theory the restriction of $\chi_{L}$ to $\mathbb{A}_{F_{0}}^{\times}$is either $\psi$ or $\psi \varepsilon_{K}$. But it cannot be $\psi \varepsilon_{K}$ : let $u=(-1,1, \ldots, 1) \in \mathbb{A}_{F_{0}, \infty}^{\times}$(where the first position corresponds to the place $v_{1}$ ). Then $\psi \varepsilon_{K}(u)=-1$, but $\chi_{L}(u)=1$ because $v_{1}$ extends to a complex embedding of $K$. Finally, (3) follows from the fact that the group $D_{8}$ has a unique 2-dimensional irreducible representation. 
Proposition 3.5. Let $\mathfrak{d}_{L / K}$ denote the discriminant of $L / K$. Then $\mathfrak{d}_{L / K}=c \cdot \mathfrak{N}_{\psi}$, where $c$ is an ideal of $F_{0}$ and $\mathfrak{N}_{\psi}$ is an ideal of $K$ such that $\mathrm{Nm}_{K / F_{0}} \mathfrak{N}_{\psi}=N_{\psi}$.

Proof. By the conductor-discriminant formula $\mathfrak{d}_{L / K}$ equals the conductor of $\chi_{L}$. Then the proposition is a consequence of the fact that the central character of $\chi_{L}$ is $\psi$, which has conductor $N_{\psi}$. Proposition 3.5 now follows from Lemma 3.6 below.

Lemma 3.6. (1) If $p \subset F_{0}$ is a prime such that $p \mid N_{\psi}$, then either $p$ splits or ramifies in $K$. In both cases, exactly one of the primes above $p$ exactly divides the conductor of $\chi_{L}$.

(2) Let $p \subset F_{0}$ be a prime such that $p \nmid N_{\psi}$ and $\mathfrak{p}^{e}$ divides exactly the conductor of $\chi_{L}$ for some prime $\mathfrak{p} \subset K$ above $p$. Then either $p$ is inert in $K$ or splits as $p \cdot R_{K}=\mathfrak{p p}^{\prime}$ and $\left(\mathfrak{p}^{\prime}\right)^{e}$ divides exactly the conductor of $\chi_{L}$.

Proof. To prove (1), let $p$ be a prime of $F_{0}$ dividing $N_{\psi}$. If $p$ splits as $\mathfrak{p p}^{\prime}$ in $K$, then by Lemma 3.4 the composition

$$
R_{0, p}^{\times} \longrightarrow R_{K, \mathfrak{p}}^{\times} \times R_{K, \mathfrak{p}^{\prime}}^{\times} \stackrel{\chi_{L, \mathfrak{p}} \cdot \chi_{L, \mathfrak{p}^{\prime}}}{\longrightarrow}\{ \pm 1\}
$$

equals $\psi_{p}$. Since by assumption $p$ does not divide 2 and $N_{\psi}$ is squarefree, $\psi_{p}$ is the unique character of order 2 of $R_{0, p}^{\times} /(1+p)$. Since $R_{K, \mathfrak{p}}^{\times} /(1+\mathfrak{p}) \simeq R_{K, \mathfrak{p}^{\prime}}^{\times} /\left(1+\mathfrak{p}^{\prime}\right) \simeq$ $R_{0, p}^{\times} /(1+p)$ we see that the character

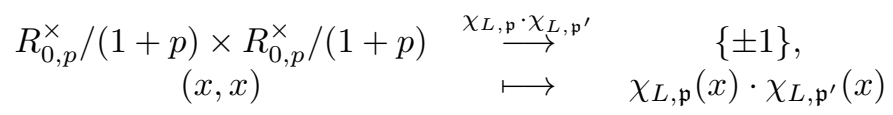

has order 2. This implies that exactly one of $\chi_{L, \mathfrak{p}}$ or $\chi_{L, \mathfrak{p}^{\prime}}$ is trivial. Suppose that $\chi_{L^{\prime}, \mathfrak{p}}$ is trivial and $\chi_{L, \mathfrak{p}}$ has order 2 . Then $\mathfrak{p}$ divides exactly the conductor of $\chi_{L}$ and $\mathfrak{p}^{\prime}$ does not divide it.

Suppose now that $p \mid N_{\psi}$ is ramified in $K$ so that $p R_{K}=\mathfrak{p}^{2}$. Then by Lemma 3.4 the composition

$$
R_{0, p}^{\times} \longrightarrow R_{K, \mathfrak{p}}^{\times} \stackrel{\chi_{L, p}}{\longrightarrow}\{ \pm 1\}
$$

equals $\psi_{p}$, which is a character of order 2 factorizing through $R_{0, p}^{\times} /(1+p)$. This implies that $\chi_{L, \mathfrak{p}}$ necessarily factorizes through $R_{K, \mathfrak{p}}^{\times} /(1+\mathfrak{p})$, because $R_{K, \mathfrak{p}}^{\times} /(1+\mathfrak{p}) \simeq$ $R_{0, p}^{\times} /(1+p)$. Therefore $\mathfrak{p}$ divides exactly the conductor $\mathfrak{d}_{L / K}$ of $\chi_{L}$.

Suppose now that $p \mid N_{\psi}$ is inert in $K$, so that $p R_{K}=\mathfrak{p}$. Again by Lemma 3.4 the character $\psi_{p}$ equals

$$
R_{0, p}^{\times} \longrightarrow R_{K, \mathfrak{p}}^{\times} \stackrel{\chi_{L, \mathfrak{p}}}{\longrightarrow}\{ \pm 1\},
$$

the composition of the natural inclusion with $\chi_{L, \mathfrak{p}}$. But the map in (19) is trivial. Indeed, in this case $\mathbb{F}_{p}^{\times}=R_{0, p}^{\times} /(1+p)$ is strictly contained in $\mathbb{F}_{\mathfrak{p}}^{\times}=R_{K, \mathfrak{p}}^{\times} /(1+\mathfrak{p})$. Then $\chi_{L, \mathfrak{p}}$ is the unique quadratic character of $\mathbb{F}_{\mathfrak{p}}^{\times}$, and such a character is always trivial on $\mathbb{F}_{p}^{\times}$. The fact that $\psi_{p}$ is trivial contradicts the fact that $p \mid N_{\psi}$, so this case does not occur.

To prove (2) we again use the fact that the localization at $p$ of the composition

$$
\mathbb{A}_{F_{0}}^{\times} \longrightarrow \mathbb{A}_{K}^{\times} \stackrel{\chi_{L}}{\longrightarrow}\{ \pm 1\}
$$

coincides with $\psi_{p}$, and therefore it is trivial because in this case $p \nmid N_{\psi}$. But $\chi_{L, \mathfrak{p}}$ has order 2, so that in particular it is not trivial. Suppose that $\chi_{L, \mathfrak{p}}$ has conductor $\mathfrak{p}^{e}$ for some $e \geqslant 1$. Observe that now, since $\mathfrak{p}$ can divide 2 , the exponent $e$ may be 
greater than 1 (in fact, it is equal to 1 except if $\mathfrak{p}$ divides 2 , in which case it may also be 2 or 3 ). In any case, the localization of (20) at $\mathfrak{p}$ is trivial only in one of the following situations:

i) The inclusion $\mathbb{A}_{F_{0}}^{\times} \longrightarrow \mathbb{A}_{K}^{\times}$localizes to a strict inclusion $R_{0, p}^{\times} /\left(1+p^{e}\right) \hookrightarrow$ $R_{K, \mathfrak{p}}^{\times} /\left(1+\mathfrak{p}^{e}\right)$.

ii) The map in (20) localizes to

$$
\begin{aligned}
& \begin{array}{ccccc}
R_{0, p}^{\times} /\left(1+p^{e}\right) & \longrightarrow & R_{0, p}^{\times} /\left(1+p^{e}\right) \times R_{0, p}^{\times} /\left(1+p^{e}\right) & \stackrel{\chi_{L, \mathfrak{p}} \cdot \chi_{L, \mathfrak{p}^{\prime}}}{\longmapsto} & \{ \pm 1\}, \\
x & \longmapsto & \longmapsto & (x, x) & \chi_{L, \mathfrak{p}}(x) \cdot \chi_{L, \mathfrak{p}^{\prime}}(x)
\end{array} \\
& \text { and } \chi_{L, \mathfrak{p}}=\chi_{L, \mathfrak{p}^{\prime}} \text {. }
\end{aligned}
$$

In the first case $p$ is inert in $K$. In the second case $p$ splits as $p \cdot R_{K}=\mathfrak{p p}^{\prime}$ and $\left(\mathfrak{p}^{\prime}\right)^{e}$ divides exactly the conductor of $\chi_{L}$.

We finally assume the following Gross-Zagier hypothesis on $E$ with respect to the ATR field extension $K / F_{0}$ :

Assumption 3.7. All prime ideals of $F_{0}$ dividing $N_{0}$ split or ramify in $K$.

In light of this assumption and the above results, we are in the setting of $\$ 2.4$ $K / F$ is an ATR extension and Assumption 2.15] is satisfied, as none of the primes dividing $N=N_{0} \operatorname{disc}\left(F / F_{0}\right)$ remain inert in $K$. Let $c \subset R_{0}$ be an integral ideal and let $R_{c}$ be the order of conductor $c$ in $R_{K}$. Let $\mathcal{O}$ be the Eichler order of level $N$ in $M_{2}\left(R_{0}\right)$ consisting of matrices which are upper triangular modulo $N$, and let $\varphi: R_{c} \hookrightarrow \mathcal{O}$ be an optimal embedding. Observe that the points $P_{\varphi}^{\beta}$ constructed in 92 are explicitly computable in this case, because Assumption 2.15 holds true. Moreover, granting Conjecture 2.14, they belong to $A\left(H_{\varphi}^{\beta}\right)$. The key point is that, as we shall see in Proposition [3.8, for suitable choices of $c$ and $\beta$ the field $M$ is contained in $H_{\varphi}^{\beta}$. Therefore, points in $E(M)$ can be constructed by projecting $P_{\varphi}^{\beta}$ via the isogeny $A \sim_{F} E^{2}$, and then taking trace over $M$.

Proposition 3.8. Let $\varphi: R_{c} \hookrightarrow \mathcal{O}$ be a normalized optimal embedding, with $c$ as in Proposition 3.5. The field $L_{\varphi}^{\beta}$ (as defined in $\$ 2.3$ ) contains $L$ if and only if $\beta_{j}(-1)=-1$ for $j=2, \ldots, r$.

Proof. First of all observe that $c$ is prime to $N$ because $\operatorname{disc}(M / F)$ is prime to $N_{E}$. Thanks to Assumption 3.7, there exists an ideal $\mathfrak{N}$ of $K$ divisible by $\mathfrak{N}_{\psi}$ and such that $\mathrm{Nm}_{K / F}(\mathfrak{N})=N$. Recall that

$$
U_{c}=\hat{R}_{0}^{\times}\left(1+c \hat{R}_{K}\right) \subset \hat{K}^{\times}
$$

and that

$$
U_{\varphi}^{+}=\left\{\beta \in U_{c} \text { such that }(\beta)_{\mathfrak{N}} \in \operatorname{ker}(\psi) \subset\left(R_{0} / N R_{0}\right)^{\times}\right\},
$$

where $\psi$ is the nebentypus of $f_{0}$ and also the character corresponding to the quadratic extension $F / F_{0}$. Here $(\beta)_{\mathfrak{N}}$ denotes the image of the local term of the idèle $\beta$ in the quotient $R_{K, \mathfrak{N}}^{\times} /\left(1+\mathfrak{N} \cdot R_{K, \mathfrak{N}}\right) \simeq\left(R_{0} / N R_{0}\right)^{\times}$. The field $L_{\varphi}^{\beta}$ is defined by

$$
\operatorname{Gal}\left(L_{\varphi}^{\beta} / K\right) \simeq \mathbb{A}_{K}^{\times} / K^{\times} U_{c}^{+} K_{\infty}^{\beta},
$$

where $K_{\infty}^{\beta}$ is as in (9). Now let $\chi_{L}: \mathbb{A}_{K}^{\times} \rightarrow\{ \pm 1\}$ be the quadratic character corresponding to $L$. Observe that by class field theory $L \subset L_{\varphi}^{\beta}$ if and only if $U_{\varphi}^{+} K_{\infty}^{\beta} \subset \operatorname{ker} \chi_{L}$. 
Let $\chi_{L}=\prod_{v} \chi_{L, v}$ be the decomposition of $\chi_{L}$ as a product of local characters. By the conductor-discriminant formula the conductor of $\chi_{L}$ is equal to $\mathfrak{d}_{L / K}=$ $c \cdot \mathfrak{N}_{\psi}$. Then $\chi_{L, f}=\prod_{\mathfrak{p} \nmid \infty} \chi_{\mathfrak{p}}$ factorizes through a character

$$
\chi_{L, f}: R_{K, c \cdot \mathfrak{N}}^{\times} /\left(1+c \cdot \mathfrak{N} R_{K, c \cdot \mathfrak{N}}\right) \rightarrow\{ \pm 1\} .
$$

First of all we check that $\chi_{L}\left(U_{\varphi}^{+} \cap R_{K, c \cdot \mathfrak{N}}^{\times}\right)=1$. Let $a=\left(a_{\mathfrak{p}}\right)_{\mathfrak{p}}$ be an element in $U_{\varphi}^{+} \cap R_{K, c \cdot \mathfrak{N}}^{\times}$. We write it as $a=a_{c} \cdot a_{\mathfrak{N}}$, where $a_{c}=\prod_{\mathfrak{p} \mid c} a_{\mathfrak{p}}$ and $a_{\mathfrak{N}}=\prod_{\mathfrak{p} \mid \mathfrak{N}} a_{\mathfrak{p}}$.

If $\mathfrak{p} \mid c$, then $\chi_{L, \mathfrak{p}}\left(a_{\mathfrak{p}}\right)=1$ by the very definition of $U_{\varphi}^{+}$. Namely, if $e=v_{\mathfrak{p}}(c)$, then $\chi_{L, \mathfrak{p}}$ has conductor $\mathfrak{p}^{e}$ so it can be regarded as a character

$$
\chi_{L, \mathfrak{p}}: R_{K, \mathfrak{p}}^{\times} /\left(1+\mathfrak{p}^{e} R_{K, \mathfrak{p}}\right) \rightarrow\{ \pm 1\} .
$$

But $a_{\mathfrak{p}}$ belongs to $\left(1+\mathfrak{p}^{e} R_{K, \mathfrak{p}}\right)$ by the definition of $U_{\varphi}^{+}$, so that $\chi_{L, p}\left(a_{\mathfrak{p}}\right)=1$. Since this is valid for any $\mathfrak{p} \mid c$ we see that $\chi_{L}\left(a_{c}\right)=1$.

Since $\mathfrak{N}$ has norm $N$ and $N$ is squarefree we have that $R_{K, \mathfrak{N}}^{\times} /\left(1+\mathfrak{N} \cdot R_{K, \mathfrak{N}}\right) \simeq$ $\left(R_{0} / N R_{0}\right)^{\times}$. Therefore the image of $a_{\mathfrak{N}}$ via the map $\mathbb{A}_{K}^{\times} \rightarrow R_{K, \mathfrak{N}}^{\times} /\left(1+\mathfrak{N} \cdot R_{K, \mathfrak{N}}\right)$ can be regarded as the image of some $b \in \mathbb{A}_{F_{0}}^{\times}$via the map $\mathbb{A}_{F_{0}}^{\times} \rightarrow \mathbb{A}_{K}^{\times} \rightarrow R_{K, \mathfrak{N}}^{\times} /(1+$ $\left.\mathfrak{N} \cdot R_{K, \mathfrak{N}}\right)$. By Lemma 3.4 we have that $\chi_{L \mid \mathbb{A}_{F_{0}}}=\psi$. Therefore, by the definition of $U_{\varphi}^{+}$we see that $\chi_{L}\left(a_{\mathfrak{N}}\right)=\psi\left(a_{\mathfrak{N}}\right)=1$.

Since we have seen that $U_{\varphi}^{+} \subseteq \operatorname{ker} \chi_{L}$, we have that $L \subseteq L_{\varphi}^{\beta}$ if and only if $\chi_{L}\left(K_{\infty}^{\beta}\right)=1$. It is clear that for the character $\beta$ such that $\beta_{j}(-1)=-1$ for $j=$ $2, \ldots, r$ this is true, because then any character of $\mathbb{A}_{K, \infty}^{\times}$is trivial when restricted to $K_{\infty}^{\beta}$. Suppose now that $\beta$ is such that $\beta_{j}(-1)=1$ for some $j$. Then the $j$-th component of $K_{\infty}^{\beta}$ is equal to $\mathbb{R}^{\times}$, and $\chi_{L}$ is not trivial restricted to this component because, by Lemma 3.3 , the field $L$ is totally imaginary so the real place $v_{j}$ extends to a complex place of $L$.

Now we let $c$ be as in Proposition [3.8, and we take $\beta: \Sigma \rightarrow\{ \pm 1\}$ to be the character such that $\beta_{j}(-1)=-1$ for $j=2, \ldots, r$. Moreover we let $\varphi: R_{c} \hookrightarrow \mathcal{O}$ be an optimal embedding normalized with respect to $\mathfrak{N}$, with $\mathfrak{N}$ an ideal of $K$ divisible by $\mathfrak{N}_{\psi}$ and of norm $N$. From now on we grant Conjecture 2.14 so that $P_{\varphi}^{\beta} \in A\left(L_{\varphi}^{\beta}\right)$. Thanks to Proposition 3.8 we can set

$$
P_{A, L}=\operatorname{Tr}_{L_{\varphi}^{\beta} / L}\left(P_{\varphi}^{\beta}\right) \in A(L) .
$$

If we denote by $C_{L}=\operatorname{rec}^{-1}\left(\operatorname{Gal}\left(L_{\varphi}^{\beta} / L\right)\right)$, then by the reciprocity law of Conjecture $2.14 P_{A, L}$ can be computed as

$$
P_{A, L}=\sum_{a \in C_{L}}\left(P_{a \star \varphi}^{\beta}\right) \in A(L) .
$$

Observe that in diagram (18) complex conjugation takes $L$ to $L^{\prime}$. Therefore the point

$$
P_{A, M}:=P_{A, L}+\overline{P_{A, L}}
$$

lies in $A(M)$. Finally, we define

$$
P_{M}=\pi\left(P_{A, M}\right) \in E(M),
$$

where $\pi: A \rightarrow E$ is the natural projection, an algebraic map defined over $F$.

Theorem 3.9. Assume Conjecture 2.14 holds true for the ATR extension $K / F$. Suppose also that the sign of the functional equation of $L(E / F, s)$ is +1 and that of $L(E / M, s)$ is -1 . Then $P_{M}$ is non-torsion if and only if $L^{\prime}(E / M, 1) \neq 0$. 
Proof. If $L^{\prime}(E / M, 1) \neq 0$, then $L(E / M, s)$ vanishes with order 1 at $s=1$. Since

$$
L(E / M, s)=L(E / F, s) L\left(E / F, \chi_{M}, s\right)
$$

it then follows that $L(E / F, 1) \neq 0$, as otherwise we would have $\operatorname{ord}_{s=1} L(E / F, s) \geqslant$ 2 , which in light of (21) is in contradiction with the fact that $\operatorname{ord}_{s=1} L(E / M, s)=1$. Then in turn we deduce from (21) that the order of vanishing of $L\left(E / F, \chi_{M}, s\right)$ is 1 at $s=1$.

By Lemma 3.4 we have $\operatorname{Ind}_{F}^{F_{0}} \chi_{M} \simeq \operatorname{Ind}_{K}^{F_{0}} \chi_{L}$, so that

$$
\begin{aligned}
L\left(E / F, \chi_{M}, s\right) & =L\left(f_{0} / F \otimes \chi_{M}, s\right)=L\left(f_{0} \otimes \operatorname{Ind}_{\mathrm{F}}^{\mathrm{F}_{0}} \chi_{\mathrm{M}}, \mathrm{s}\right) \\
& =L\left(f_{0} \otimes \operatorname{Ind}_{\mathrm{K}}^{\mathrm{F}_{0}} \chi_{\mathrm{L}}, \mathrm{s}\right)=L\left(f_{0} / K, \chi_{L}, s\right),
\end{aligned}
$$

and therefore $L\left(f_{0} / K, \chi_{L}, s\right)$ vanishes with order 1 at $s=1$. If we denote by $\chi: \operatorname{Gal}\left(L_{\varphi}^{\beta} / K\right) \rightarrow \mathbb{C}$ the character $\chi_{L}$ viewed as a character of $\operatorname{Gal}\left(L_{\varphi}^{\beta} / K\right)$, then part (2) of Conjecture 2.14 implies that the point

$$
P_{\chi}=\sum_{\sigma \in \operatorname{Gal}\left(L_{\varphi}^{\beta} / K\right)} \chi(\sigma)^{-1} \sigma\left(P_{\varphi}^{\beta}\right) \in A\left(L_{\varphi}^{\beta}\right)
$$

is non-torsion.

In order to apply the reciprocity law, let us view for a moment the fields $K, L$ and $L_{\varphi}^{\beta}$ as subfields of $\mathbb{C}$ via a place of $\overline{\mathbb{Q}}$ extending $v_{j}$, for a fixed $j \in\{2, \ldots, r\}$. Since $K$ is real under $v_{j}$ and $L$ is complex, we see that complex conjugation induces an element in $s \in \operatorname{Gal}\left(L_{\varphi}^{\beta} / K\right)$ that restricts to a generator of $\operatorname{Gal}(L / K)$. But $s$ corresponds under the reciprocity map to the idèle

$$
\xi_{j}:=\left(\xi_{\infty}, \xi_{f}\right)=(1, \ldots, 1, \stackrel{j)}{-1}, 1, \ldots, 1) \times(1,1, \ldots) \in K_{\infty}^{\times} \times \hat{K}^{\times},
$$

so by part (3) of 2.14 we have that $s\left(P_{\varphi}^{\beta}\right)=\beta\left(\xi_{\infty}\right) P_{\varphi}^{\beta}=-P_{\varphi}^{\beta}$. Then we have that

$$
\begin{aligned}
P_{\chi} & =\sum_{\sigma \in \operatorname{Gal}\left(L_{\varphi}^{\beta} / L\right)} \sigma\left(P_{\varphi}^{\beta}\right)+\sum_{\sigma \in \operatorname{Gal}\left(L_{\varphi}^{\beta} / L\right)} \chi(\sigma s) \sigma s\left(P_{\varphi}^{\beta}\right) \\
& =\sum_{\sigma \in \operatorname{Gal}\left(L_{\varphi}^{\beta} / L\right)} \sigma\left(P_{\varphi}^{\beta}\right)+\sum_{\sigma \in \operatorname{Gal}\left(L_{\varphi}^{\beta} / L\right)} \chi_{L}(s) \sigma\left(-P_{\varphi}^{\beta}\right) \\
& =2 \cdot \operatorname{Tr}_{L_{\varphi}^{\beta} / L}\left(P_{\varphi}^{\beta}\right)=2 \cdot P_{A, L},
\end{aligned}
$$

which implies that $P_{A, L}$ is non-torsion. Moreover, as $s\left(P_{\varphi}^{\beta}\right)=-P_{\varphi}^{\beta}$ we have that $P_{A, L} \in A(L)^{\chi_{L}}$. Then $P_{A, M}=P_{A, L}+\overline{P_{A, L}}$ belongs to $A(M)$ and is non-torsion as well. Since the projection $\pi: A \rightarrow E$ is defined over $F$ and $A(M) \simeq E^{2}(M)$, we see that $P_{M}=\pi\left(P_{A}(M)\right)$ belongs to $E(M)$ and is of infinite order.

Let $W_{N}$ denote the Atkin-Lehner involution on $S_{2}\left(\Gamma_{\psi}(N)\right)$ corresponding to the ideal $N$. By abuse of notation we also denote by $W_{N}$ the involution that it induces on $A$. Then the splitting of the variety $A$ over $F$ is accomplished by the action of $W_{N}$. More precisely we have that

$$
A \sim_{F}\left(1+W_{N}\right) A \times\left(1-W_{N}\right) A .
$$

Let $\lambda_{N}$ be the pseudoeigenvalue of $f_{0}$ corresponding to $N$; that is, the complex number satisfying that $W_{N}\left(f_{0}\right)=\lambda_{N} \cdot \bar{f}_{0}$. Observe that the modular form

$$
\alpha_{f_{0}}^{\beta}:=\frac{1}{1+\lambda_{N}}\left(f_{0}+W_{N}\left(f_{0}\right)\right)
$$


is normalized. In view of Conjecture 2.8 the lattice of $E$ can be computed as

$$
\Lambda_{E}=\left(\Omega_{2}^{-} \cdots \Omega_{r}^{-}\right)^{-1} \cdot\left\langle\int_{Z} \alpha_{f_{0}}^{\beta}\right\rangle
$$

where $Z \in H_{2}\left(X_{\psi}(\mathbb{C}), \mathbb{Z}\right)$ runs over the cycles such that $\int_{Z}\left(\omega_{f_{0}}^{\beta}-W_{N}\left(\omega_{f_{0}}^{\beta}\right)\right)=0$. From this we obtain the following explicit analytic formula for the points $P_{M}$.

Theorem 3.10. Let

$$
J_{\mathcal{M}}=\left(\Omega_{2}^{-} \cdots \Omega_{r}^{-}\right)^{-1} \cdot\left(\sum_{a \in C_{L}} \int_{\tilde{T}_{a \star \varphi}} \alpha_{f_{0}}^{\beta}\right) .
$$

Then the point $P_{M}$ can be computed as

$$
P_{M}=\eta\left(J_{\mathcal{M}}+\bar{J}_{\mathcal{M}}\right),
$$

where $\eta$ is the Weierstrass parametrization $\eta: \mathbb{C} / \Lambda_{E} \rightarrow E(\mathbb{C})$ and the bar denotes complex conjugation.

Proof. The Atkin-Lehner involution $W_{N}$ is defined over $F$ and $F L=\mathcal{M}$, so $\eta\left(J_{\mathcal{M}}\right)$ belongs to $E(\mathcal{M})$. We recall that we are viewing $\mathcal{M}$ as a subfield of $\mathbb{C}$ by means of $v_{1}$. Under this embedding $\mathcal{M}$ is complex and $M$ is real, and therefore

$$
P_{M}=\operatorname{Tr}_{\mathcal{M} / M}\left(\eta\left(J_{\mathcal{M}}\right)\right)=\eta\left(J_{\mathcal{M}}\right)+\overline{\eta\left(J_{\mathcal{M}}\right)} .
$$

Since $E$ is defined over $F$ and $F \subseteq \mathbb{R}$ we have that the Weierstrass map commutes with complex conjugation, and (26) follows.

Remark 3.11. Observe that $W_{N}\left(P_{L}\right)=\operatorname{Tr}_{L^{\prime \beta} / L^{\prime}}\left(W_{N}\left(P_{\varphi}^{\beta}\right)\right)$ belongs to $A\left(L^{\prime}\right)$. Since complex conjugation does not fix $K$, by part (3) of Conjecture 2.14 we see that

$$
\overline{P_{A, L}}=W_{N}\left(\sigma\left(P_{A, L}\right)\right)+P_{t}
$$

for some $\sigma \in \operatorname{Gal}(L / K)$ and some $P_{t} \in A\left(L^{\prime}\right)_{\text {tors. }}$. If $\sigma$ turns out to be trivial and $P_{t}$ belongs to $A\left(F_{0}\right)_{\text {tors }}$, then the point $P_{A, L}+W_{N}\left(P_{A, L}\right)$ is already defined over $M$. In this case $\eta\left(J_{\mathcal{M}}\right)$ lies in $E(M)$ and $P_{M}$ coincides, up to torsion, with $2 \cdot \eta\left(J_{\mathcal{M}}\right)$. As we will see, this is the situation encountered in the example of 3.3 .

Remark 3.12. Observe that the integral appearing in the formula of Theorem 3.10 is completely explicit. Indeed, in the case where $f_{0}$ has trivial nebentypus, an algorithm for determining the chains $\tilde{T}_{\varphi}$ is worked out in [DL], based on the approach taken in Das2. As we showed in $\$ 2.4$. Darmon-Logan's method adapts to provide an explicit description of $\tilde{T}_{\varphi}$ also in the current setting, in which $f_{0}$ has quadratic nebentypus.

3.2. Comparison with Gartner's ATC points. Let us keep the notation of the previous $\$ 3.1$ in particular $E / F$ is an elliptic curve defined over the totally real field $F$ and $M / F$ is an ATC quadratic extension. The curve $E$ is modular: its isogeny class corresponds by the Eichler-Shimura construction to the Hilbert modular form $f$ that one obtains from $f_{0}$ by base-change to $F$ in such a way that

$$
L(E, s)=L(f, s)
$$

as in (5).

We place ourselves under the hypothesis of Theorem 3.9 so that we assume $N_{E}$ is squarefree, the sign of the functional equation of $L(E / F, s)$ is +1 and that of $L(E / M, s)$ is -1 . 
As discussed in 91 our point $P_{M}$ in $E(M)$ is expected to coexist with another point $P_{M}^{\mathrm{Gar}}([\mathrm{Ga2}, \S 5.4])$, provided Conjecture 2.14 for the abelian extensions of $M$ holds true. This point can be manufactured by applying the machinery of $\S \$ 2.1$, 2.2. 2.3. setting $M / F$ to play the role of the extension $K / F_{0}$ of loc. cit.

Let us sketch the details: let $B$ be the quaternion algebra over $F$ which ramifies precisely at all the archimedean places of $F$ except the two places extending $v_{1}$, and at the prime ideals $\wp \mid N_{E}$ that remain inert in $M$. That this is a set of even cardinality is guaranteed by the sign of the functional equation of $L(E / M, s)$. Let $\mathcal{O}$ be an Eichler order in $B$ of squarefree level, divisible exactly by those primes $\wp \mid N_{E}$ which split or ramify in $M$.

Let $R_{M}$ denote the ring of integers of $M$ and fix a normalized optimal embedding $\varphi_{M} \in \mathcal{E}\left(R_{M}, \mathcal{O}\right)$. In the notation of $\S \$ 2.1$ and 2.2 we have $r=2$ and $\Sigma=\{ \pm 1\}$. Take $\beta$ to be the trivial character and, granting Conjecture 2.14 let $P_{\varphi} \in E\left(L_{\varphi}^{\beta}\right)$ denote the Darmon point associated with this choice. Set

$$
P_{M}^{\mathrm{Gar}}=\operatorname{Tr}_{L_{\varphi}^{\beta} / M}\left(P_{\varphi}^{\beta}\right) \in E(M) .
$$

It is expected that the Néron-Tate height of $P_{M}^{\text {Gar }}$ should be related to $L^{\prime}(E / M, 1)$, while the Néron-Tate height of $P_{M}$ constructed in this paper should be connected to $L^{\prime}\left(E / F, \chi_{M}, 1\right)$. Hence from the basic quality,

$$
L^{\prime}(E / M, 1)=L(E / F, 1) L^{\prime}\left(E / F, \chi_{M}, 1\right),
$$

we propose the following conjecture about the relation between $P_{M}$ and $P_{M}^{\mathrm{Gar}}$. Let

$$
\Omega_{E / F}=\frac{\prod_{\tau: F \hookrightarrow \mathbb{R}} c_{E^{\tau}}}{\sqrt{\operatorname{disc}(F)}}
$$

where $c_{E^{\tau}}$ is either the real period or twice the real period of $E^{\tau}=E \times_{\tau} \mathbb{R}$, depending on whether or not $E^{\tau}(\mathbb{R})$ is connected.

Conjecture 3.13. The point $P_{M}^{\mathrm{Gar}}$ is of infinite order if and only if $P_{M}$ is of infinite order and $L(E / F, 1) \neq 0$. Moreover,

$$
P_{M}^{\mathrm{Gar}}=2^{s} \ell \cdot P_{M},
$$

where $s$ is an integer which depends on $M$ and $\ell \in \mathbb{Q}^{\times}$satisfies $\ell^{2}=\frac{L(E / F, 1)}{\Omega_{E / F}}$.

Remark 3.14. At the moment, the only instance in which the rationality of StarkHeegner points is proven unconditionally is the one considered by Bertolini and Darmon in $\mathrm{BD}$, in which they prove a $p$-adic analogue of Conjecture 3.13 over genus fields. In that setting the Stark-Heegner points are constructed by means of $p$-adic periods, while in the present article the points are constructed using complex periods. This is a key difference, and the circle of ideas used by Bertolini and Darmon does not seem to adapt well to the complex setting.

To better explain the difference between both settings, let us place ourselves in a very particular case of Conjecture 3.13 set $F_{0}=\mathbb{Q}$ and let $f_{0} \in S_{2}(N, \varepsilon)$ be a classical eigenform of prime level $N \equiv 1(\bmod 4)$, quadratic nebentypus character $\varepsilon$ and whose Fourier coefficients generate an imaginary quadratic field. Set $F=\mathbb{Q}(\sqrt{N})$ and let $E / F$ be the $\mathbb{Q}$-curve associated to $f_{0}$ by the Eichler-Shimura construction. Finally, let $f$ denote the Doi-Naganuma lift of $f_{0}$ to $F$ : it is a Hilbert modular form of level 1 over $F$. Note that in this particular scenario our constructions and conjectures are exactly the ones considered in [DRZ]. 
The basic relation between $f_{0}$ and its base change $f$ is

$$
L(f, s)=L\left(f_{0}, s\right) \cdot L\left(\bar{f}_{0}, s\right),
$$

where $\bar{f}_{0}$ denotes the eigenform obtained from $f_{0}$ by complex conjugating of its Fourier coefficents. Then (28) and its counterpart for twists by Dirichlet characters can be used to prove that the elliptic curves $\mathbb{C} / \Lambda_{f}$ and $\mathbb{C} / \Lambda_{f_{0}}$ are isogenous (see [Od, §17] for the details).

Under suitable sign hypotheses, Conjecture 3.13 predicts that a certain ATR point $P_{M}^{?}$ in $\mathbb{C} / \Lambda_{f}$ is a simple multiple of a Heegner point $P_{M} \in E(M) \subset E(\mathbb{C}) \simeq$ $\mathbb{C} / \Lambda_{f_{0}}$. This conjecture is motivated by the equality

$$
L^{\prime}\left(f, \chi_{M}, 1\right)=L^{\prime}\left(f_{0} \otimes \chi_{L}, 1\right) .
$$

However, in the complex setting the derivative $L^{\prime}\left(f_{0} \otimes \chi_{L}, 1\right)$ is only expected to capture the height of $P_{M}$, but falls short of recovering the point $P_{M}$ itself. A similar remark holds for the ATR point $P_{M}^{?}$.

In the $p$-adic setting of $[\mathrm{BD}$, the $p$-adic avatar of both sides of (29) are played by the special values (and not the derivative) of suitable $p$-adic $L$-functions at a point outside the region of interpolation. These special values are proved to retrieve the (formal logarithm of the) Heegner and Stark-Heegner points themselves, and not just their height. This is the fundamental fact that allows Bertolini and Darmon to prove their main result in $\mathrm{BD}$, and also the reason why a similar proof does not immediately generalize to the scenario considered in this note.

3.3. A numerical example. In this section we give the details for the computation of an ATC point on a particular elliptic curve. We used Sage [S+09] for all the numerical calculations. We begin by describing the elliptic curve and the corresponding Hilbert modular form $f_{0}$, which we will take to be the base change of a modular form $f$ over $\mathbb{Q}$.

3.3.1. The curve and the modular form. Let $f$ be the (unique up to Galois conjugation) classical newform over $\mathbb{Q}$ of level 40 and nebentypus $\varepsilon(\cdot)=\left(\frac{10}{.}\right)$. It corresponds to the third form of level 40 in the table 4.1 of the appendix to $\mathrm{Qu}$. We see from this table that the modular abelian variety $A_{f}$ has dimension 4 . Moreover, it breaks (up to isogeny) into the fourth power of an elliptic curve $E / F$, where $F=\mathbb{Q}(\sqrt{2}, \sqrt{5})$. Jordi Quer computed an equation for $E$ using the algorithms of GL]; a global minimal model of $E$ is given by

$$
y^{2}+b_{1} x y+b_{3} y=x^{3}+b_{2} x^{2}+b_{4} x+b_{6},
$$

where

$$
\begin{aligned}
& b_{1}=1-9 / 2 \sqrt{2}+3 \sqrt{5}-1 / 2 \sqrt{10} \\
& b_{2}=-15 / 2+13 / 2 \sqrt{2}-9 / 2 \sqrt{5}+5 / 2 \sqrt{10} \\
& b_{3}=-11 / 2-27 / 2 \sqrt{2}+17 / 2 \sqrt{5}+3 / 2 \sqrt{10} \\
& b_{4}=41 / 2+8 \sqrt{2}-15 / 2 \sqrt{5}-8 \sqrt{10} \\
& b_{6}=525 / 2+8 \sqrt{2}-13 / 2 \sqrt{5}-84 \sqrt{10}
\end{aligned}
$$

Let $F_{0}=\mathbb{Q}(\sqrt{2})$ and let $v_{1}$ (resp. $v_{2}$ ) be the embedding taking $\sqrt{2}$ to the positive (resp. negative) square root of 2 . Since $E$ is a $\mathbb{Q}$-curve, it is also an $F_{0}$-curve. If we set $\alpha=\sqrt{10}+\sqrt{5}+\sqrt{2}$, then $M=F(\sqrt{\alpha})$ is an ATC extension of $F$. Since the conductor of $E / F$ is equal to 1 , the sign of the functional equation of $L(E / F, s)$ is 
+1 and the sign of $L(E / M, s)$ is -1 . The point $P_{\mathrm{nt}} \in E(M)$ whose $x$ coordinate is given by

$x=\frac{-3259+2126 \sqrt{\alpha}-8957 \sqrt{\alpha}^{2}+5297 \sqrt{\alpha}^{3}-4989 \sqrt{\alpha}^{4}+1954 \sqrt{\alpha}^{5}-743 \sqrt{\alpha}^{6}+39 \sqrt{\alpha}}{72}$

is a generator of the Mordell-Weil group of $E(M)$. Theorem 3.9 predicts that the point $P_{M}$ coincides, up to torsion, with a multiple of $P_{\mathrm{nt}}$. We computed an approximation to $J_{\mathcal{M}} \in \mathbb{C} / \Lambda_{E}$ with an accuracy of 30 decimal digits using formula (25). Let $J_{\mathrm{nt}} \in \mathbb{C} / \Lambda_{E}$ be a preimage of $P_{\mathrm{nt}}$ by Weierstrass's uniformization map. Then the relation

$$
7 \cdot J_{\mathcal{M}}-14 \cdot J_{\mathrm{nt}} \in \Lambda_{E}
$$

holds up to the computed numerical precision of 30 digits. The torsion group $E(M)_{\text {tors }}$ is isomorphic to $\mathbb{Z} / 14 \mathbb{Z}$. Observe that this gives numerical evidence for the fact that $\eta\left(J_{M}\right)$ is already a non-torsion point in $E(M)$ in this case. We find a similar relation for $P_{M}=\eta\left(J_{\mathcal{M}}+\overline{J_{\mathcal{M}}}\right)$ :

$$
7 \cdot\left(J_{\mathcal{M}}+\overline{J_{\mathcal{M}}}\right)-28 \cdot J_{\mathrm{nt}} \in \Lambda_{E} .
$$

In the rest of the section we provide the details about the computation of $J_{\mathcal{M}}$, beginning with those related to compute the Hilbert modular form attached to $E$ over $F_{0}$.

Let $f_{0}$ be the base change of $f$ to $F_{0}$. Denote by $N$ the level of $f$, and let $A=$ $\operatorname{Res}_{F / F_{0}} E$, which is a $\mathrm{GL}_{2}$-variety over $F_{0}$. By Milne's formula Mi2, Proposition 1] it has conductor cond $\left(A / F_{0}\right)=(25)$. By the Shimura-Taniyama conjecture for $\mathrm{GL}_{2}$-type varieties $A$ is isogenous to $A_{f_{0}}$, which has conductor $N^{2}$. Then we see that $N=(5)$ and that $f_{0}$ belongs to $S_{2}\left(\Gamma_{\psi}(N)\right)$, where $\psi$ is the restriction of $\varepsilon$ to $\operatorname{Gal}\left(\overline{\mathbb{Q}} / F_{0}\right)$. By identifying $\varepsilon$ with a character $\mathbb{A}_{\mathbb{Q}}^{\times} \rightarrow\{ \pm 1\}$ by means of class field theory, $\psi$ can be identified with the idèle character $\varepsilon \circ \mathrm{Nm}_{F_{0} / \mathbb{Q}}: \mathbb{A}_{F_{0}}^{\times} \rightarrow\{ \pm 1\}$.

The Fourier coefficients of $f=\sum_{n \geqslant 1} c_{n} q^{n}$ can be explicitly computed in Sage. Let us see how to compute the coefficients of $f_{0}$ in terms of the $c_{n}$ 's. The field $E_{f}=\mathbb{Q}\left(\left\{c_{n}\right\}\right)$ turns out to be $\mathbb{Q}(\sqrt{2}, \sqrt{-3})$. Let $\operatorname{Gal}\left(E_{f} / \mathbb{Q}\right)=\{1, \sigma, \tau, \sigma \tau\}$, where $\sigma$ denotes the automorphism that fixes $\mathbb{Q}(\sqrt{-3})$ and $\tau$ the one that fixes $\mathbb{Q}(\sqrt{2})$. The inner twists of $f$ are given by

$$
\chi_{\sigma}=\varepsilon_{\mathbb{Q}(\sqrt{5})}, \chi_{\tau}=\varepsilon_{\mathbb{Q}(\sqrt{10})}, \chi_{\sigma \tau}=\chi_{\sigma} \chi_{\tau}=\varepsilon_{\mathbb{Q}(\sqrt{2})},
$$

where $\varepsilon_{\mathbb{Q}(\sqrt{a})}$ denotes the Dirichlet character corresponding to $\mathbb{Q}(\sqrt{a}) / \mathbb{Q}$. Recall that inner twists are defined by the relations $f^{\rho}=\chi_{\rho} \otimes f$, for $\rho \in \operatorname{Gal}\left(E_{f} / \mathbb{Q}\right)$. This is also equivalent to saying that $c_{p}^{\rho}=\chi_{\rho}(p) c_{p}$ for all primes $p$ not dividing the level of $f$ (see [Ri] for more details).

Lemma 3.15. $L\left(f_{0}, s\right)=L(f, s) L\left(f^{\sigma \tau}, s\right)$.

Proof. Indeed $f_{0}$ is the base change of $f$ to $F_{0}=\mathbb{Q}(\sqrt{2})$. Then,

$$
L(f, s)=L(f, s) L\left(f \otimes \varepsilon_{\mathbb{Q}(\sqrt{2})}, s\right)=L(f, s) L\left(f \otimes \chi_{\sigma \tau}, s\right)=L(f, s) L\left(f^{\sigma \tau}, s\right) .
$$

The $L$-series of $f_{0}$ is of the form

$$
L\left(f_{0}, s\right)=\prod_{\mathfrak{p} \nmid N}\left(1-a_{\mathfrak{p}} \operatorname{Nm}(\mathfrak{p})^{-s}+\psi(\mathfrak{p}) \operatorname{Nm}(\mathfrak{p})^{1-2 s}\right)^{-1} \prod_{\mathfrak{p} \mid N}\left(1-a_{\mathfrak{p}} \operatorname{Nm}(\mathfrak{p})^{-s}\right)^{-1},
$$

for some coefficients $a_{\mathfrak{p}}$, indexed by the primes in $F_{0}$. 
Lemma 3.16. Let $\mathfrak{p}$ be a prime in $F_{0}$, and let $p=\mathfrak{p} \cap \mathbb{Z}$. Then

$$
a_{\mathfrak{p}}= \begin{cases}c_{p} & \text { if } \varepsilon_{\mathbb{Q}(\sqrt{2})}(p)=1 \text { and } p \neq 5, \\ c_{p}^{2}-2 \varepsilon(p) p & \text { if } \varepsilon_{\mathbb{Q}(\sqrt{2})}(p)=-1 \text { and } p \neq 5, \\ c_{p}^{2} & \text { if } p=5, \\ c_{p}+c_{p}^{\sigma \tau} & \text { if } p=2 .\end{cases}
$$

Proof. For a rational prime $p$ let $L_{p}\left(f_{0}, s\right)=\prod_{\mathfrak{p} \mid p} L_{\mathfrak{p}}(f, s)$ denote the product of local factors for the primes $\mathfrak{p} \mid p$. For $p \neq 2,5$ Lemma 3.15 gives that

$$
L_{p}\left(f_{0}, s\right)=\left(1-c_{p} p^{-s}+\varepsilon(p) p^{1-2 s}\right)^{-1}\left(1-c_{p}^{\sigma \tau} p^{-s}+\varepsilon(p) p^{1-2 s}\right)^{-1} .
$$

If $\varepsilon_{\mathbb{Q}(\sqrt{2})}(p)=1$, then $p$ splits in $F_{0}$ so there are two primes $\mathfrak{p}_{1}, \mathfrak{p}_{2}$ dividing $p$, each one having norm $p$. On the other hand, $c_{p}^{\sigma \tau}=c_{p}$ (because $\chi_{\sigma \tau}=\varepsilon_{\mathbb{Q}(\sqrt{2})}(p)$ ) and $\psi\left(\mathfrak{p}_{i}\right)=\varepsilon\left(\operatorname{Nm}\left(\mathfrak{p}_{i}\right)\right)=\varepsilon(p)$. Comparing (33) and (32) we see that $a_{\mathfrak{p}_{i}}=c_{p}$.

If $\varepsilon_{\mathbb{Q}(\sqrt{2})}(p)=-1$, then there is only one prime $\mathfrak{p}$ dividing $p$, and $\psi(\mathfrak{p})=$ $\varepsilon(\operatorname{Nm}(\mathfrak{p}))=1$. On the other hand $c_{p}^{\sigma \tau}=-c_{p}$, so

$$
\begin{aligned}
L_{p}\left(f_{0}, s\right) & =\left(1-c_{p} p^{-s}+\varepsilon(p) p^{1-2 s}\right)^{-1}\left(1+c_{p} p^{-s}+\varepsilon(p) p^{1-2 s}\right)^{-1} \\
& =1+2 \varepsilon(p) p^{1-2 s}-c_{p}^{2} p^{-2 s}+p^{2-4 s} \\
& =1+\left(2 \varepsilon(p) p-c_{p}^{2}\right) \operatorname{Nm}(\mathfrak{p})^{-s}+\operatorname{Nm}(\mathfrak{p})^{1-2 s}
\end{aligned}
$$

and we see that $a_{p}=c_{p}^{2}-2 \varepsilon(p) p$.

If $p=5$, then $\chi_{\sigma \tau}(p)=-1$, so $c_{p}^{\sigma \tau}=-c_{p}$. Since 5 divides the level of $f$ we have that

$$
L_{p}\left(f_{0}, s\right)=\left(1-c_{p} p^{-s}\right)^{-1}\left(1+c_{p} p^{-s}\right)^{-1}=\left(1-c_{p}^{2} \mathrm{Nm}(\mathfrak{p})^{-s}\right)^{-1},
$$

so that $a_{\mathfrak{p}}=c_{p}^{2}$.

Finally, if $p=2$, then $(p)=\mathfrak{p}^{2}$ in $F_{0}$. But $\mathfrak{p}$ does not divide the level of $f_{0}$ and $\psi(\mathfrak{p})=-1$ (because $\mathfrak{p}$ is inert in $F$ ), so $L_{\mathfrak{p}}\left(f_{0}, s\right)=L_{p}\left(f_{0}, s\right)$ is of the form

$$
L_{\mathfrak{p}}\left(f_{0}, s\right)=\left(1-a_{\mathfrak{p}} p^{-s}-p^{1-2 s}\right)^{-1} .
$$

On the other hand, $p$ divides the level of $f$, so that

$$
L_{\mathfrak{p}}\left(f_{0}, s\right)=\left(1-c_{p} p^{-s}\right)\left(1-c_{p}^{\sigma \tau} p^{-s}\right)=\left(1-\left(c_{p}+c_{p}^{\sigma \tau}\right) p^{-s}+c_{p} c_{p}^{\sigma \tau} p^{-2 s}\right) .
$$

It turns out that $c_{p} c_{p}^{\sigma \tau}=-p$, so (34) and (35) match and we see that $a_{\mathfrak{p}}=$ $c_{p}+c_{p}^{\sigma \tau}$.

3.3.2. Computation of the ATC point. Let $e=\sqrt{2}-1$ be a fundamental unit of $F_{0}$. Observe that $e_{1}=v_{1}(e)>0$ and $e_{2}=v_{2}(e)<0$. Let $\beta:\{ \pm 1\} \rightarrow\{ \pm 1\}$ be the non-trivial character. The differential $\omega_{f_{0}}^{\beta}$ is then the one corresponding to

$$
\omega_{f_{0}}^{\beta}=\frac{-4 \pi^{2}}{\sqrt{8}}\left(f_{0}\left(z_{1}, z_{1}\right) d z_{1} d z_{2}-f_{0}\left(e_{1} z_{1}, e_{2} \bar{z}_{2}\right) d\left(e_{1} z_{1}\right) d\left(e_{2} \bar{z}_{2}\right)\right) .
$$

As for $W_{N}\left(\omega_{f_{0}}^{\beta}\right)$, it is easy to compute because $W_{N}\left(f_{0}\right)=\lambda_{N} \bar{f}_{0}$, where the pseudoeigenvalue $\lambda_{N}$ is equal to $a_{(N)} / N=\frac{-1+2 \sqrt{-6}}{5}$. Therefore

$$
W_{N}\left(\omega_{f_{0}}^{\beta}\right)=\frac{(4-8 \sqrt{-6}) \pi^{2}}{5 \sqrt{8}}\left(\bar{f}_{0}\left(z_{1}, z_{1}\right) d z_{1} d z_{2}-\bar{f}_{0}\left(e_{1} z_{1}, e_{2} \bar{z}_{2}\right) d\left(e_{1} z_{1}\right) d\left(e_{2} \bar{z}_{2}\right)\right),
$$

and we have completely determined $\alpha_{f_{0}}^{\beta}=\frac{1}{1+\lambda_{N}}\left(\omega_{f_{0}}^{\beta}+W_{N}\left(\omega_{f_{0}}^{\beta}\right)\right.$. 
Recall that $M$ is not Galois over $F_{0}$ and that the diagram of subfields of its Galois closure $\mathcal{M}$ is the one given in (18). The ATR field $K$ is easily computed to be $K=F_{0}(\omega)$, where $\omega^{2}+(\sqrt{2}+1) \omega+3 \sqrt{2}+4=0$. Here we remark that $K$ is complex under the embeddings extending $v_{1}$, and it is real under the embeddings extending $v_{2}$. The discriminant of $L / K$ is an ideal $\mathfrak{N}$ which in this case satisfies $\mathrm{Nm}_{K / F_{0}}(\mathfrak{N})=N$. Therefore the ideal $c$ of Proposition 3.8 is equal to 1 for this example. Let $\varphi: R_{K} \hookrightarrow \mathcal{O}$ be the optimal embedding of the maximal order $R_{K}$ into the Eichler order of conductor $N$ of $M_{2}\left(F_{0}\right)$ given by

$$
\varphi(\omega)=\left(\begin{array}{cc}
-\sqrt{2}+2 & -2 \\
5 & -3
\end{array}\right) .
$$

By Proposition 3.8 we see that $L$ is contained in $L_{\varphi}^{\beta}$, but $L_{\varphi}^{\beta}$ is a quadratic extension of the narrow Hilbert class field of $K$. Since $K$ turns out to have narrow class number 1 , we see that $L_{\varphi}^{\beta}$ is a quadratic extension of $K$, and hence equals $L$. This means that $H_{\varphi}^{\beta}=\mathcal{M}$, so that according to Conjecture 2.14 the point $P_{\varphi}^{\beta}$ is defined over $\mathcal{M}$.

The fixed point of $K^{\times}$under $\varphi$ (with respect to $v_{1}$ ) is

$$
z_{1} \simeq 0.358578643762691+0.520981147679366 \cdot i .
$$

The unit

$$
e_{K}=(-10 \sqrt{2}+14) w+7 \sqrt{2}-11
$$

satisfies that $\mathrm{Nm}_{K / F_{0}}\left(e_{K}\right)=1$ and generates the group of such units, so that

$$
\gamma_{\varphi}=\varphi\left(e_{K}\right)=\left(\begin{array}{ll}
-27 \sqrt{2}+37 & 20 \sqrt{2}-28 \\
-50 \sqrt{2}+70 & 37 \sqrt{2}-53
\end{array}\right)
$$

and

$$
\gamma_{\varphi} \cdot \infty=\frac{-27 \sqrt{2}+37}{-50 \sqrt{2}+70}=\frac{4 \sqrt{2}+11}{10} .
$$

To compute $J_{\mathcal{M}}$ we need to evaluate the 3 -limit integral

$$
J_{\mathcal{M}}=\int^{z_{1}} \int_{\infty}^{\gamma_{\varphi} \cdot \infty} \alpha_{f_{0}}^{\beta}=\int^{z_{1}} \int_{\infty}^{\frac{4 \sqrt{2}+11}{10}} \alpha_{f_{0}}^{\beta} .
$$

The next step is to use properties (13), (14), and (15) to transform (36) into a sum of the usual 4-limit integrals, because they can be numerically computed by integrating (a truncation of) the Fourier series of $\alpha_{f_{0}}^{\beta}$. Observe that $\alpha_{f_{0}}^{\beta}$ is invariant under $W_{N}=W_{(5)}$, so we have the following additional invariance property:

$$
\int^{x} \int_{y}^{z} \alpha_{f_{0}}^{\beta}=\int^{\frac{-1}{5 x}} \int_{\frac{-1}{5 y}}^{\frac{-1}{5 z}} \alpha_{f_{0}}^{\beta} .
$$

We will also use the following matrices, both belonging to $\Gamma_{\psi}(N)$ :

$$
G=\left(\begin{array}{cc}
4 \sqrt{2}+11 & -3 \sqrt{2}+5 \\
10 & -6 \sqrt{2}+9
\end{array}\right), \quad H=\left(\begin{array}{cc}
-15 \sqrt{2}+21 & -\sqrt{2}-1 \\
-35 \sqrt{2}+50 & 1
\end{array}\right) .
$$


Since $\gamma_{\varphi} \cdot \infty=G \cdot \infty$ and $G \cdot 0=\frac{-3 \sqrt{2}+5}{-6 \sqrt{2}+9}=\sqrt{2} / 3+1$, we have that

$$
\begin{aligned}
\int^{z_{1}} \int_{\infty}^{\gamma_{\varphi} \cdot \infty} \alpha_{f_{0}}^{\beta} & =\int^{z_{1}} \int_{\infty}^{G \cdot \infty} \alpha_{f_{0}}^{\beta}=\int^{z_{1}} \int_{\infty}^{G \cdot 0} \alpha_{f_{0}}^{\beta}+\int^{z_{1}} \int_{G \cdot 0}^{G \cdot \infty} \alpha_{f_{0}}^{\beta} \\
& =\int^{z_{1}} \int_{\infty}^{\sqrt{2} / 3+1} \alpha_{f_{0}}^{\beta}+\int^{G^{-1} \cdot z_{1}} \int_{0}^{\infty} \alpha_{f_{0}}^{\beta} .
\end{aligned}
$$

Now, since $H \cdot \infty=\frac{-15 \sqrt{2}+21}{-35 \sqrt{2}+50}=\frac{-3 \sqrt{2}}{10}$ and $H \cdot 0=-\sqrt{2}-1$ we have that

$$
\begin{aligned}
& \int^{z_{1}} \int_{\infty}^{\sqrt{2} / 3+1} \alpha_{f_{0}}^{\beta}=\int^{z_{1}-1} \int_{\infty}^{\sqrt{2} / 3} \alpha_{f_{0}}^{\beta}=\int^{\frac{-1}{5\left(z_{1}-1\right)}} \int_{0}^{\frac{-3 \sqrt{2}}{10}} \alpha_{f_{0}}^{\beta} \\
& =\int^{\frac{-1}{5\left(z_{1}-1\right)}} \int_{0}^{H \cdot \infty} \alpha_{f_{0}}^{\beta}=\int^{\frac{-1}{5\left(z_{1}-1\right)}} \int_{0}^{H \cdot 0} \alpha_{f_{0}}^{\beta}+\int^{\frac{-1}{5\left(z_{1}-1\right)}} \int_{H \cdot 0}^{H \cdot \infty} \alpha_{f_{0}}^{\beta} \\
& =\int^{\frac{-1}{5\left(z_{1}-1\right)}} \int_{0}^{-\sqrt{2}-1} \alpha_{f_{0}}^{\beta}+\int^{H^{-1} \cdot \frac{-1}{5\left(z_{1}-1\right)}} \int_{0}^{\infty} \alpha_{f_{0}}^{\beta} \\
& =\int^{\frac{-1}{5\left(z_{1}-1\right)}} \int_{0}^{\infty} \alpha_{f_{0}}^{\beta}+\int^{\frac{-1}{5\left(z_{1}-1\right)}} \int_{\infty}^{-\sqrt{2}-1} \alpha_{f_{0}}^{\beta}+\int^{H^{-1} \cdot \frac{-1}{5\left(z_{1}-1\right)}} \int_{0}^{\infty} \alpha_{f_{0}}^{\beta} \\
& =\int^{\frac{-1}{5\left(z_{1}-1\right)}} \int_{0}^{\infty} \alpha_{f_{0}}^{\beta}+\int^{\frac{-1}{5\left(z_{1}-1\right)}+\sqrt{2}+1} \int_{\infty}^{0} \alpha_{f_{0}}^{\beta}+\int^{H^{-1} \cdot \frac{-1}{5\left(z_{1}-1\right)}} \int_{0}^{\infty} \alpha_{f_{0}}^{\beta} \\
& =\int_{\frac{-1}{5\left(z_{1}-1\right)}+\sqrt{2}+1}^{\frac{-1}{5\left(z_{1}-1\right)}} \int_{0}^{\infty} \alpha_{f_{0}}^{\beta}+\int^{H^{-1} \cdot \frac{-1}{5\left(z_{1}-1\right)}} \int_{0}^{\infty} \alpha_{f_{0}}^{\beta} .
\end{aligned}
$$

Now, putting together (38) and (39) we have that

$$
\begin{aligned}
& \int^{z_{1}} \int_{\infty}^{\gamma_{\varphi} \cdot \infty} \alpha_{f_{0}}^{\beta}=\int^{G^{-1} \cdot z_{1}} \int_{0}^{\infty} \alpha_{f_{0}}^{\beta}+\int_{\frac{-1}{5\left(z_{1}-1\right)}+\sqrt{2}+1}^{\frac{-1}{5\left(z_{1}-1\right)}} \int_{0}^{\infty} \alpha_{f_{0}}^{\beta}+\int^{H^{-1} \cdot \frac{-1}{5\left(z_{1}-1\right)}} \int_{0}^{\infty} \alpha_{f_{0}}^{\beta} \\
& =\int^{\frac{-1}{5 G^{-1} \cdot z_{1}}} \int_{\infty}^{0} \alpha_{f_{0}}^{\beta}+\int_{\frac{-1}{5\left(z_{1}-1\right)}+\sqrt{2}+1}^{\frac{-1}{5\left(z_{1}-1\right)}} \int_{0}^{\infty} \alpha_{f_{0}}^{\beta}+\int^{H^{-1} \cdot \frac{-1}{5\left(z_{1}-1\right)}} \int_{0}^{\infty} \alpha_{f_{0}}^{\beta} \\
& =\int_{\frac{-1}{5\left(z_{1}-1\right)}+\sqrt{2}+1}^{\frac{-1}{5\left(z_{1}-1\right)}} \int_{0}^{\infty} \alpha_{f_{0}}^{\beta}+\int_{\frac{-1}{5 G^{-1} \cdot z_{1}}}^{H^{-1} \cdot \frac{-1}{5\left(z_{1}-1\right)}} \int_{0}^{\infty} \alpha_{f_{0}}^{\beta} .
\end{aligned}
$$

Now both of these integrals can be computed easily, because for $x, y \in \mathcal{H}$ one has that

$$
\int_{x}^{y} \int_{0}^{\infty}=\int_{x}^{y} \int_{0}^{i / \sqrt{5}}+\int_{x}^{y} \int_{i / \sqrt{5}}^{\infty}=\int_{\frac{-1}{5 x}}^{\frac{-1}{5 y}} \int_{\infty}^{i / \sqrt{5}}+\int_{x}^{y} \int_{i / \sqrt{5}}^{\infty},
$$

which are integrals with all of their limits lying in $\mathcal{H}$, and they can be computed by integrating the Fourier expansion term by term.

Let $\Lambda_{1}$ and $\Lambda_{2}$ be the period lattices of $E$ with respect to $v_{1}$ and $v_{2}$, and denote by $\Omega_{1}^{+}, \Omega_{2}^{+}$the real periods and by $\Omega_{1}^{-}, \Omega_{2}^{-}$the imaginary periods. Using the above 
limits we integrated the truncation of the Fourier expansion of $\alpha_{f_{0}}^{\beta}$ up to ideals of norm 160000 obtaining

$$
\begin{aligned}
J_{\mathcal{M}}=\left(\Omega_{2}^{-}\right)^{-1} \int^{z_{1}} \int_{\infty}^{\gamma_{\varphi} \infty} \alpha_{f_{0}}^{\beta} & \simeq 6.1210069519472105302223690235 \\
& +i \cdot 5.4381903029486320686211994460
\end{aligned}
$$

Recall that $J_{\mathrm{nt}}$ stands for the logarithm of $P_{\mathrm{nt}}$ in $\mathbb{C} / \Lambda_{E}$. The actual value is

$$
J_{\mathrm{nt}} \simeq 3.3835055058970249460140888086+i \cdot 2.7190951514743160343105997232 .
$$

We have that

$$
7 \cdot J_{\mathcal{M}}-14 \cdot J_{\mathrm{nt}}+\Omega_{1}^{+} \simeq 3.742356 \cdot 10^{-27}-i \cdot 3.23117 \cdot 10^{-27},
$$

which is the numerical evidence for the fact that relation (31) holds and that, up to torsion, $\eta\left(J_{\mathcal{M}}\right)$ equals $2 P_{\mathrm{nt}}$.

\section{ACKNOWLEDGEMENTS}

We are thankful to Jordi Quer for computing the equation of the elliptic curve used in 3.3 .1 for us. We are also thankful to Jan Nekovár and Jérôme Gärtner for pointing out some inaccuracies in an earlier version of the article, and to the anonymous referee for many useful comments and suggestions which improved the exposition. The first author wants to thank the Max Planck Institute for Mathematics for their hospitality and financial support during his stay at the Institute, where part of the present work was carried out. The first and second authors received financial support from DGICYT Grant MTM2009-13060-C02-01 and from 2009 SGR 1220.

\section{REFERENCES}

[BD] M. Bertolini, H. Darmon, The rationality of Stark-Heegner points over genus fields of real quadratic fields, Ann. Math. 170 (2009), 343-369. MR2521118(2010m:11072)

[BCDT] C. Breuil, B. Conrad, F. Diamond, and R. Taylor, On the modularity of elliptic curves over Q: wild 3-adic exercises, J. Amer. Math. Soc. 14 (2001), no.4, 843-939. MR1839918 (2002d:11058)

[BR] D. Blasius, J. D. Rogawski, Motives for Hilbert modular forms, Inventiones Math. 114 (1993), no.1, 55-87. MR1235020 (94i:11033)

[Da1] H. Darmon, Rational points on modular elliptic curves, CBMS Regional Conference Series in Mathematics 101, 2003. MR2020572 (2004k:11103)

[Da2] H. Darmon, Integration on $\mathcal{H}_{\ell} \times \mathcal{H}$ and arithmetic applications, Ann. Math. 154 (2001), no. 3, 589-639. MR1884617 (2003j:11067)

[Das1] S. Dasgupta, Stark-Heegner points on modular Jacobians, Ann. Sci. École Normale Supérieure, 4e sér. 38 (2005), no.3, 427-469. MR2166341(2006e:11080)

[Das2] S. Dasgupta, Gross-Stark Units, Stark-Heegner Points, and Class Fields of Real Quadratic Fields, Ph.D. Thesis, University of California-Berkeley (2004). MR2706449

[De] L. Dembele, An algorithm for modular elliptic curves over real quadratic fields, Experiment. Math. 17 (2008), no.4, 427-438. MR.2484426 (2010a:11119)

[Di] L.V. Dieulefait, Langlands Base Change for GL(2), Annals of Math., to appear.

[DL] H. Darmon, A. Logan, Periods of Hilbert modular forms and rational points on elliptic curves, Int. Math. Res. Not. 40 (2003), 2153-2180. MR1997296 (2005f:11110) 
[DRZ] H. Darmon, V. Rotger, Y. Zhao, The Birch and Swinnerton-Dyer conjecture for $\mathbb{Q}$-curves and Oda's period relations, Proc. Int. Symp. in honor of T. Oda, Series on Number Theory and its Applications 7, Y. Hamahata, T. Ichikawa, A. Murase, T. Sugano, eds., World Scientific (2012), 1-40. MR2908033

[DV] L. Dembélé, J. Voight, Explicit methods for Hilbert modular forms, in H. Darmon, F. Diamond, L.V. Dieulefait, B. Edixhoven, V. Rotger (eds.), Elliptic curves, Hilbert modular forms and Galois deformations, Birkhauser, Centre de Recerca Matemàtica (Bellaterra, Spain), to appear.

[Ga1] J. Gartner, Points de Darmon et varieties de Shimura, Ph.D. thesis, Jussieu (2011), available at http://jerome.gaertner.free.fr/

[Ga2] J. Gartner, Darmon's points and quaternionic Shimura varieties, Canad. J. Math. 64 (2012), 1248-1288. MR 2994664

[Gr] M. Greenberg, Stark-Heegner points and the cohomology of quaternionic Shimura varieties, Duke Math. J. 147 (2009), no.3, 541-575. MR2510743 (2010f:11097)

[GSS] M. Greenberg, M. A. Seveso, S. Shahabi, p-adic L-functions, p-adic Jacquet-Langlands, and arithmetic applications, preprint 2011.

[GZ] B.H. Gross and D.B. Zagier, Heegner points and derivatives of L-series. Invent. Math. 84 (1986), no.2, 225-320. MR833192 (87j:11057)

[GL] J. González, J-C. Lario, $\mathbb{Q}$-curves and their Manin ideals, Amer. J. Math. 123 (2001), no.3, 475-503. MR.1833149 (2002e:11070)

[GM1] X. Guitart, M. Masdeu, Computation of ATR Darmon points on non-geometrically modular elliptic curves, Experimental Mathematics 22(1):85-98 (2013). MR3038785

[GM2] X. Guitart, M. Masdeu, Elementary matrix decomposition and the computation of Darmon points with higher conductor, to appear in Mathematics of Computation.

[Ko] V.A. Kolyvagin, Finiteness of $E(\mathbf{Q})$ and $\mathbb{L}(E, \mathbf{Q})$ for a subclass of Weil curves, Izv. Akad. Nauk SSSR Ser. Mat. 52 (1988), no.3, 670-671; translation in Math. USSR-Izv. 32 (1989), no.3, 523-541. MR954295 (89m:11056)

[LRV] M. Longo, V. Rotger, S. Vigni, On rigid analytic uniformizations of Jacobians of Shimura curves, to appear in American J. Math.

[LV] M. Longo, S. Vigni, The rationality of quaternionic Darmon points over genus fields of real quadratic fields, preprint 2011.

[Mi1] J. S. Milne, Introduction to Shimura varieties, available at http://www.jmilne.org/math.

[Mi2] J. S. Milne, On the arithmetic of abelian varieties, Invent. Math. 178 (2009), no.3, 485-504. MR0330174(48:8512)

[MS] Y. Matsushima, G. Shimura, On the cohomology groups attached to certain vector valued differential forms on the product of the upper half planes, Ann. Math. (2) 78 (1963), 417449. MR0155340 (27:5274)

[Od] T. Oda, Periods of Hilbert modular surfaces. Progress in Mathematics 19, Birkhäuser, Boston, Mass. 1982. MR670069 (83k:10057)

[Qu] J. Quer, Fields of definition of building blocks, Math. Comp. 78 (2009), 537-554. Appendix available at http://arxiv.org/abs/1202.3061 MR.2448720 (2010a:11109)

[Ri] K. A. Ribet, Twists of modular forms and endomorphisms of abelian varieties, Math. Ann. 253 (1980), no.1, 43-62. MR594532(82e:10043)

$\left[\mathrm{S}^{+} 09\right]$ W. A. Stein et al., Sage Mathematics Software (Version 4.7), The Sage Development Team, 2011, http://www.sagemath.org.

[Sh] G. Shimura, The special values of the zeta functions associated with Hilbert modular forms, Duke Math. J., 45 (1978), no.3, 637-679. MR507462 (80a:10043)

[SW] C. Skinner, A. Wiles, Nearly ordinary deformations of irreducible residual representations, Ann. Fac. Sci. Toulouse Math. (6) 10 (2001), 185-215. MR1928993(2004b:11073)

[Vi] M. F. Vigneras, Arithmétique des algèbres de quaternions. Lecture Notes in Mathematics 800, Springer, Berlin 1980. MR580949 (82i:12016)

[Wi] A. Wiles, Modular elliptic curves and Fermat's last theorem, Ann. Math. (2) 141 (1995), no.3, 443-551. MR 1333035 (96d:11071)

[Yo] H. Yoshida, On the zeta functions of Shimura varieties and periods of Hilbert modular forms. Duke Math. J. 75 (1994), no. 1, 121-191. MR1284818 (95d:11059) 
[Zh] S.-W. Zhang, Arithmetic of Shimura curves, Sc. China Math. 53 (2010), no.3, 573-592. MR2608314(2011b:11084)

Max-Planck-Institute for Mathematics, Vivatsgasse 7, 53111 Bonn, Germany - And - Departament de Matemàtica Aplicada II, Universitat Politècnica de Catalunya, C. Jordi Girona 1-3, 08034 Barcelona, Spain

E-mail address: xevi.guitart@gmail.com

Current address: Institut für Experimentelle Mathematik, Universität Duisburg-Essen, Ellernstr. 29, 45326, Essen, Germany

Departament de Matemàtica Aplicada II, Universitat Politècnica de Catalunya, C. Jordi Girona 1-3, 08034 Barcelona, Spain

E-mail address: victor.rotger@upc.edu

Department of Mathematics, John Abbott College, Montreal, Quebec, Canada H9X 3L9

E-mail address: yu.zhao@johnabbott.qc.ca 